\title{
Chondroitin Sulfate Elicits Systemic Pathogenesis In Mice By Interfering With Gut Microbiota Homeostasis
}

\author{
Tao Liao ${ }^{1,3}$, Yan-Ping Chen ${ }^{1,3}$, Shui-Qing Huang ${ }^{4}$, Li-Li Tan ${ }^{1}$, Chang-Qing Li ${ }^{1}$, Xin-An \\ Huang ${ }^{1}$, Qin Xu' ${ }^{1}$, Qi Wang ${ }^{2 * *}$, Qing-Ping Zeng ${ }^{1 * *}$ \\ ${ }^{1}$ Tropical Medicine Institute, Guangzhou University of Chinese Medicine, Guangzhou 510405, China. \\ ${ }^{2}$ Clinical Pharmacology Institute, Guangzhou University of Chinese Medicine, Guangzhou 510405 China. \\ ${ }^{3}$ Basic Medical Science College, Guangzhou University of Chinese Medicine, Guangzhou 510405, \\ China. \\ ${ }^{4}$ These authors contributed equally. \\ ${ }^{* *}$ Correspondence: qpzeng@gzucm.edu.cn; wangq@gzucm.edu.cn
}

\section{SUMMARY}

Whether chondroitin sulfate (CS), a common ingredient naturally occurring in livestock and poultry products, improves osteoarthritis remains debating. Here, we show for the first time that CS induces steatogenesis, atherogenesis, and dementia-like pathogenesis in mice. Gut microbiome analysis revealed the sulfatase-secreting bacteria Rikenella and the sulfate-reducing bacteria Desulfovibrio are enriched. Surprisingly, berberine use boosts CS-induced multi-loci inflammatory manifestations by further increasing the abundance of Rikenella and Desulfovibrio, whereas cephalosporin reinforces the colon mucus barrier via flourishing Akkermansia muciniphila and upregulating mucin expression. Mechanistically, berberine aggravates mucus lining injury by prompting mucin degradation, endotoxin leakage, neutralizing antibody induction, pro-inflammatory cytokine burst, lactic acid accumulation and energy currency depletion in multiple organs and tissues. Taken together, CS evokes the early-phase pathogenesis toward steatohepatitis, atherosclerosis, and dementia upon augmenting gut opportunistic infection, and a sustained antibiotic monotherapy does not deprive the risk of CS-driven systemic inflammatory disorders.

\section{INTRODUCTION}

Chondroitin sulfate (CS), a common biochemical ingredient in livestock and poultry products, is prescribed as a drug for treatment of osteoarthritis (OA) in 22 countries, including the European Union, based on the efficacy and safety data available from clinical trials (Vergés et al., 2004). Nevertheless, CS is still regulated in the United States as a dietary supplement. In 2004, the Food and Drug Administration (FDA) denied a petition that requests $C S$ be labeled as reducing the risk of $O A$ and OA-related joint pain, tenderness, and swelling (FDA, 2004). A systematic review summarizing 20 clinical trials concluded the symptomatic benefit of CS from the large-scale and methodologically sound trials is minimal or nonexistent, so CS use in the routine clinical practice should be 
bioRxiv preprint doi: https://doi org/10.1101/142588; this version posted May 27, 2017. The copyright holder for this preprint (which was not certified by peer review) is the author/funder, who has granted bioRxiv a license to display the preprint in perpetuity. It is made available under aCC-BY-NC-ND 4.0 International license.

discouraged (Reichenbach et al., 2007). However, a contrast conclusion was drawn that compelling evidence supports CS interferes with OA progression (Clegg et al., 2006; Reginster et al., 2007).

An updated Cochrane review on the clinical trials involving CS indicated most are of low quality, but there is some evidence of short-term improvement in pain and few side effects; it does not appear to improve or maintain the health of affected joints (Singh et al., 2015). it was warned that CS should not be used to treat patients with symptomatic knee OA because of failure in relief of the condition (Jevseva et al., 2013). It was also demonstrated that a combined therapy of CS with glucosamine sulfate or glucosamine hydrochloride does not improve joint damage in a rabbit model of knee OA (Roman-Blas et al., 2017a), and CS plus glucosamine sulfate shows no superiority over placebo in a randomized, double-blind and placebo-controlled clinical trial in patients with knee OA (Roman-Blas et al., 2017b). In contrast, an anti-inflammatory effect of CS was filed in amelioration of some inflammatory diseases, at least including inflammatory bowel disease (IBD) (Segarra et al., 2016; Holleran et al., 2017).

While CS is helpful to OA remains controversial, the inconsistent results regarding the "beneficial" role of CS in OA might be argued not to be attributed to the "bad" design of experiments. Actually, there are challenges against contamination of CS with heparin, which may cause some adverse clinical events, such as allergy and hypertension (Guerrini et al., 2008; Kishimoto et al., 2008). However, no reports was noted to concern a potential effect of the individual gut microbiota on the differential pharmaceutical outcome of CS. Although evidence on CS-driven inflammatory pathogenesis by interfering with the gut microbiota is still lacking, an implication of the gut microbiota disturbance has been addressed in atherosclerosis (Spence, 2016; Wilson et al., 2016), non-alcoholic fatty liver disease (NAFLD) (Mokhtari et al., 2017), non-alcoholic steatohepatitis (NASH) (Brandl \& Schnabl, 2017), and cardiovascular diseases (CVD) (Lippi et al., 2017; Garcia-Rios et al., 2017).

As a common ingredient in livestock and poultry products, CS was recognized to increase the abundance of Bacteroides thetaiotaomicron, a species of sulfatase-secreting bacteria (SSB) that degrades the glycoprotein-rich dietary sulfate and colon mucus, which supply the sulfate to Desulfovibrio piger, a sulfate-reducing bacteria (SRB) (Rey et al., 2013), arising a possibility of CS-driven coordinated overgrowth of SSB and SRB as a food chain. Indeed, many species of CS-degrading bacteria, including $B$. thetaiotaomicron $\mathrm{J} 1$ and $82, B$. ovitus $\mathrm{E} 3$ and Clostridium hathewayi $\mathrm{R} 4$, were isolated from healthy subjects (Shang et al., 2016). Alternatively, heme as a common component in the red meat was found to increase the abundance of Akkermansia muciniphila, a mucindegrading and mucus-damaging commensal gut microbiota, which can facilitate the aberrant colon epithelial proliferation by exploiting the mucin-containing carbohydrates and amino acids as substrate for growth, and also by employing the mucin-derived sulfate as an electron acceptor to give rise to sulfide and sulfur dioxide (ljssennager et al., 2015).

Considering CS as a sulfate is similar with mucin, a typical protein constituent of the mucus layer overlying the gut epithelium (Johansson et al., 2013), we suggest herein a hypothesis of "organ-specific anti- or pro-inflammation" followed by CS-induced SSB overgrowth, mucin degradation and endotoxin leakage. In a more detail, CS promotes 
SSB overgrowth, and then SSB degrade the O-glycon-containing mucin, hence opening the mucin-crossing mucus barrier, and leaking the bacterial endotoxin lipopolysaccharide (LPS) into the blood. The circulated LPS would elicit production of neutralizing antibody and secretion of pro-inflammatory cytokines to scavenge LPS, during which organs without LPS might show an anti-inflammatory feature, whereas organs with LPS might exhibit a pro-proinflammatory character. Of course, CS either ameliorates or aggravates OA might be also dependent on the individual gut microbiota status.

We fed mice with $25 \mathrm{mg}$ of CS daily to induce gut opportunistic infection, and also used $100 \mathrm{mg} / \mathrm{ml}$ berberine (BER) or $100 \mathrm{mg} / \mathrm{ml}$ cephalosporin (CEF) to control gut opportunistic infection. BER is considered antibiotic (Li \& Zuo, 2010) albeit with a low bioavailability (Liu et al., 2016), so it should directly and non-selectively kill gut bacteria. Upon fed CS combined with or without BER or CEF, we disclosed for the first time that CS not only flourishes SSB and interferes with gut microbiota homeostasis, but also induces the multiple inflammatory pathogenesis, such as steatogenesis, atherogenesis, and dementia-like amyloidosis and cognitive deficits. Surprisingly, BER use for one month was noticed to aggravate the multiple pathogenic alterations by increasing the richness of SSB. It could be anticipated that the astonishing achievements regarding CS- and BER/CEF-exerted dual effects of anti- and pro-inflammation should pave a path toward a thorough elucidation of the rationale underlying gut opportunistic infection-originated inflammatory disorders and metabolic syndromes.

\section{RESULTS}

\section{CS Alters Gut Microbiota Communities And BER/CEF Interferes With Gut Microbiota} Homeostasis

To assess the effect of animal-based diets on the gut microbiota homeostasis, we fed mice with CS or lard (LD) and performed the 16S VX region-based gut microbiome analysis (Additional file 1). As results, all tested mice show two major gut microbiota phyla among Top 10 abundant phyla: Firmicutes and Bacteroidetes. As illustrated in Figure 1A, CS nourishes more Firmicutes and less Bacteroidetes, whereas LD flourishes more Bacteroidetes and less Firmicutes. By comparison with Top 35 abundant genera, CS mice exhibit 12 abundant genera (red color) within Firmicutes and two abundant genera within Proteobacteria, whereas LD mice possess two abundant genera within Firmicutes and one abundant genus within Actinobacteria (Figure 1B). As indicated, while Bacteroides are bile tolerant bacteria that adapt to high-fat diets, Firmicutes are polysaccharide degradable bacteria that adapt to high-fiber diets (David et al. 2014).

It is obviously that CS also increases an abundance of the harmful Escherichia-shigella, whereas LD allows an overgrowth of the beneficial Bicidobacterium. Additionally, Top 100 operational taxonomic units (OTUs) are more similar between AL.NC1 and CS.TT1 than other comparisons because they share 58 common OTUs (Figure 1C), suggesting LD causes more gut microbiome changes than CS. According to the evolutionary tree constructed from Top 100 abundant genera, it was distinguishable that Lactobacillus is the most abundant genus in both CS and LD mice, and Allobacterium and Alloprevotella are the most abundant genera in AL mice (Figure 1D). 
To evaluate the effects of antibacterial agents on gut microbiota changes, we treated CS/LD mice with BER or CEF and monitored gut microbiome changes. As compared with CS.TT1, BER significantly increases the abundance of Firmicutes in CS.BER2, whereas CEF sightly increases the abundance of Firmicutes in CS.CEF3 (Figure 1E). In contrast, while BER slightly increases the richness of Bacteroidetes, CEF significantly increases the richness of Bacteroidetes. Surprisingly, BER enriches Desulfovibrio, as seen from CS.BER2, and CEF flourishes A. muciniphila, as seen from LD.CEF3 (Figure 1F). It was observed that 171 common OTUs are shared among AL.NC1, CS.TT1, CS.BER2, and CS.CEF3 (Figure 1G). On the other hand, it was also noticed that there are 134 common OTUs among AL.NC1, LD.TT1, LD.BER2, and LD.CEF3 (Figure 1H). As considering the $\beta$-diversity that represents the proportion between regional and local species diversity, it was seen that AL.NC1 has the least difference from CS.TT1, but the most difference from LD.CEF3 (Figure 1I).

Our determination revealed CS enriches $53 \%$ of Bacteroidetes and $42 \%$ of Firmicutes. In similar, LD enriches $65 \%$ of Bacteroidetes and $33 \%$ of Firmicutes. These results addressed livestock and poultry-sourced CS and LD allow the overgrowth of Bacteroidetes. Other authors also indicated an animal-based high fat/high protein food intake can increase the abundance of bile tolerant Bacteroides, Alistips and Bilophila, whereas a plant-based high fiber food intake can increase the richness of polysaccharide degradable Firmicutes and Prevotella (Wu et al., 2011; David et al., 2014; KovatchevaDatchary et al., 2015).

\section{CS/LD And BER/CEF Enrich Different Bacterial Genera/Species Belonging To SRB}

\section{And SSB}

To investigate the difference and extent of CS/LD and BER/CEF in enriching SRB and SSB, we particularly analyzed the proportion of Desulfovibrio, Rikenella, and A. muciniphila in the Kingdom of Bacteria (Table 1). It was noticeable that CS increases the abundance of Desulfovibrio from $0.006 \%$ ( $2 \%$ in the Phylum of Proteobacteria) in AL.NC1 (Figure 2A) to $0.70 \%$ (92\% in Proteobacteria) in CS.TT1 (Figure 2B), accounting for more than 100 -fold increases. While BER further increases its abundance to $3.00 \%$ (74\% in Proteobacteria) in CS.BER2 (Figure 2C), CEF decreases its abundance to $0.003 \%$ ( $40 \%$ in Proteobacteria) in CS.CEF3 (Figure 2D). It was also seen from Table 1 that CS increases the abundance of Rikenella from $0.001 \%$ in AN.NC1 to $0.05 \%$ in CS.TT1, estimating up to 50 -fold increases. BER further increases its abundance to $0.70 \%$ in CS.BER2, whereas CEF decreases its abundance to 0 in CS.CEF.

In the LD groups, it was observed that LD, BER or CEF does not support the growth of Desulfovibrio, which is undetectable in LD.TT1, LD.BER2 and LD.CEF3 (Table 1). As to Rikenella, its abundance is increased from $0.001 \%$ in AL.NC1 to $0.40 \%$ in LD.TT1, accounting for an increase of 400 folds, suggesting LD prompts the growth of Desulfovibrio. Regarding the effect of antibiotics, it was measured that LD.BER2 harbors $0.40 \%$ of Desulfovibrio, whereas LD.CEF3 decreases its abundance to 0 , indicating CEF rather than BER can eradicate Desulfovibrio (Table 1). A similar trend was also observed in the CS group, where BER increases the abundance of Desulfovibrio, while CEF decreases its abundance. 
bioRxiv preprint doi: https://doi org/10 1101/142588; this version posted May 27 2017. The copyright holder for this preprint (which was not certified by peer review) is the author/funder, who has granted bioRxiv a license to display the preprint in perpetuity. It is made available under aCC-BY-NC-ND 4.0 International license.

A

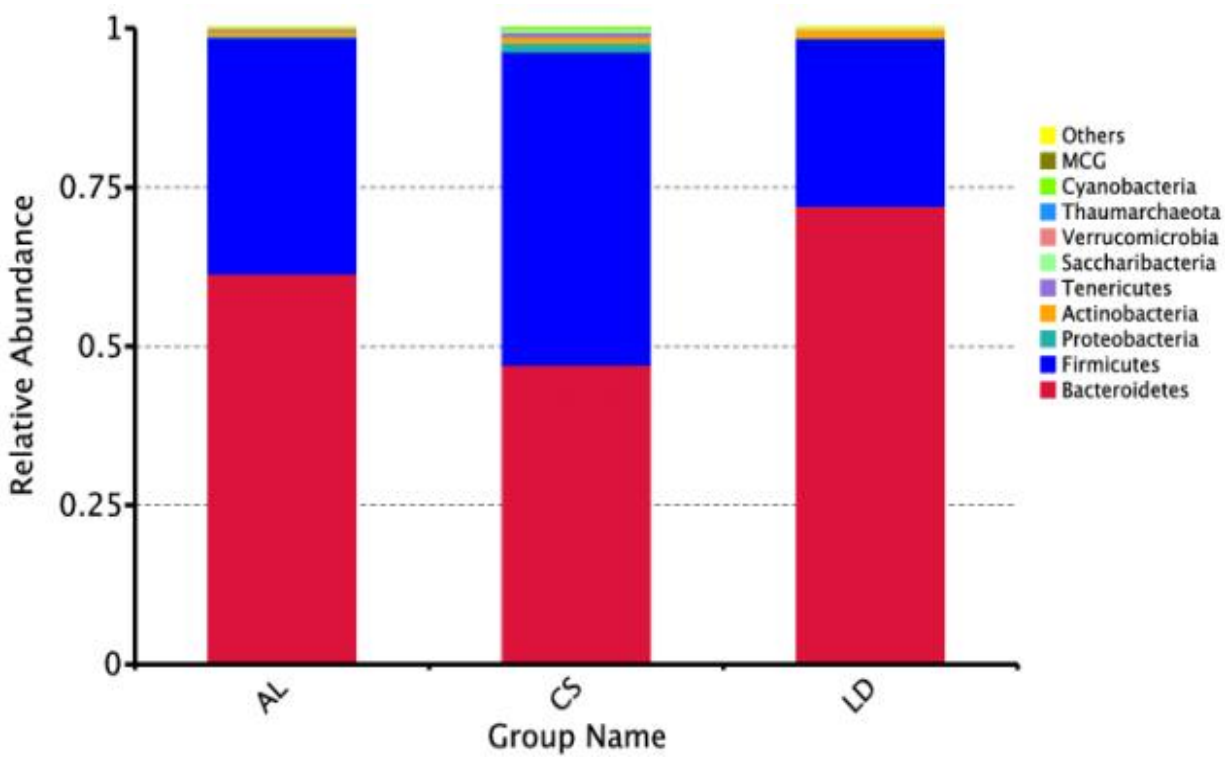

B

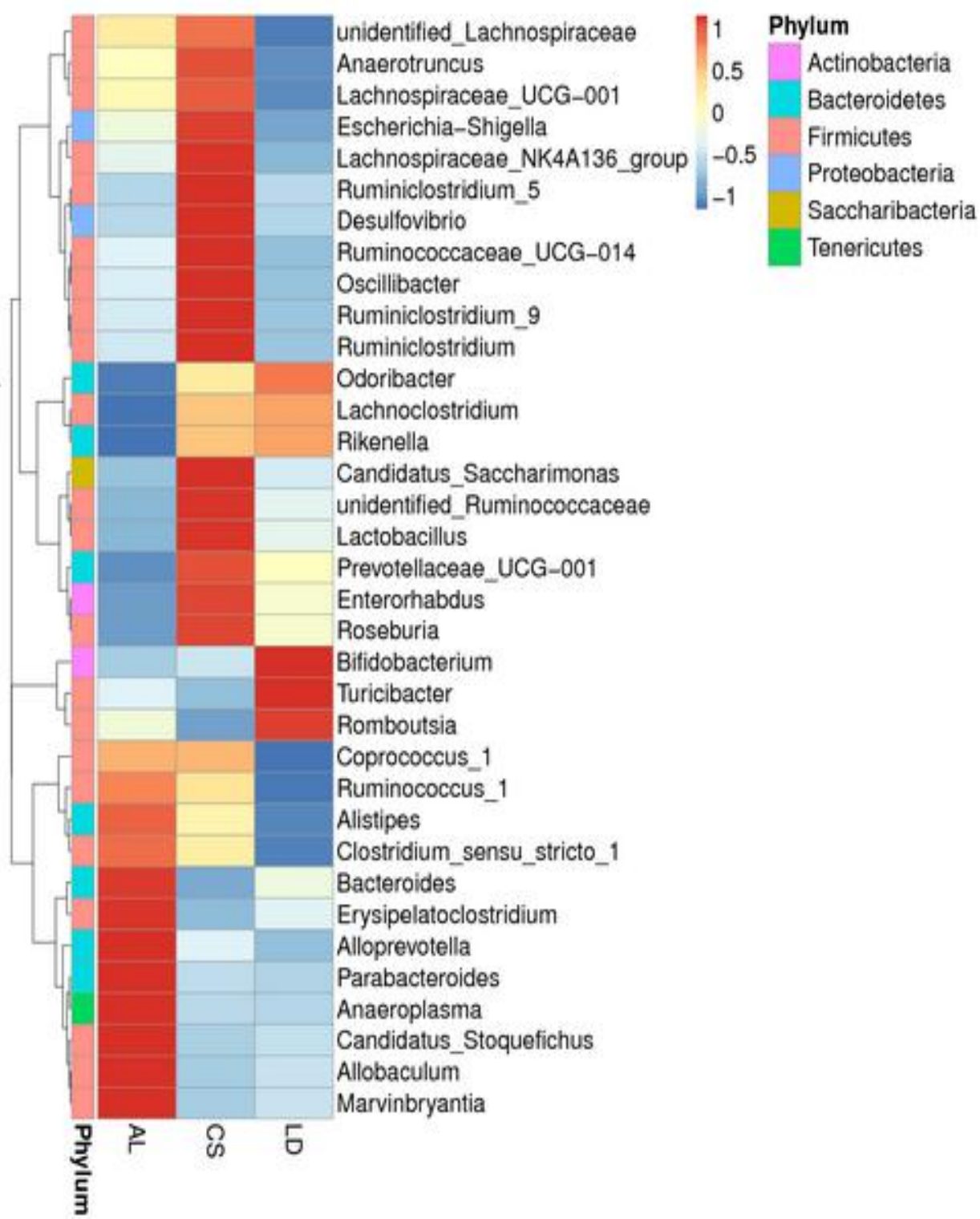


bioRxiv preprint doi: https://doi org/10.1101/142588; this version posted May 27, 2017. The copyright holder for this preprint (which was not certified by peer review) is the author/funder, who has granted bioRxiv a license to display the preprint in perpetuity. It is made available under aCC-BY-NC-ND 4.0 International license.

C

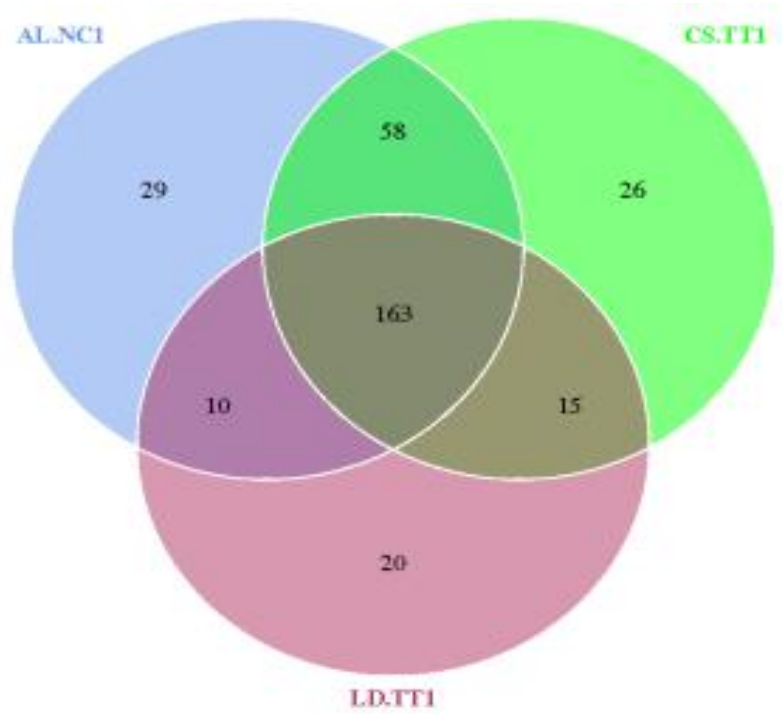

D

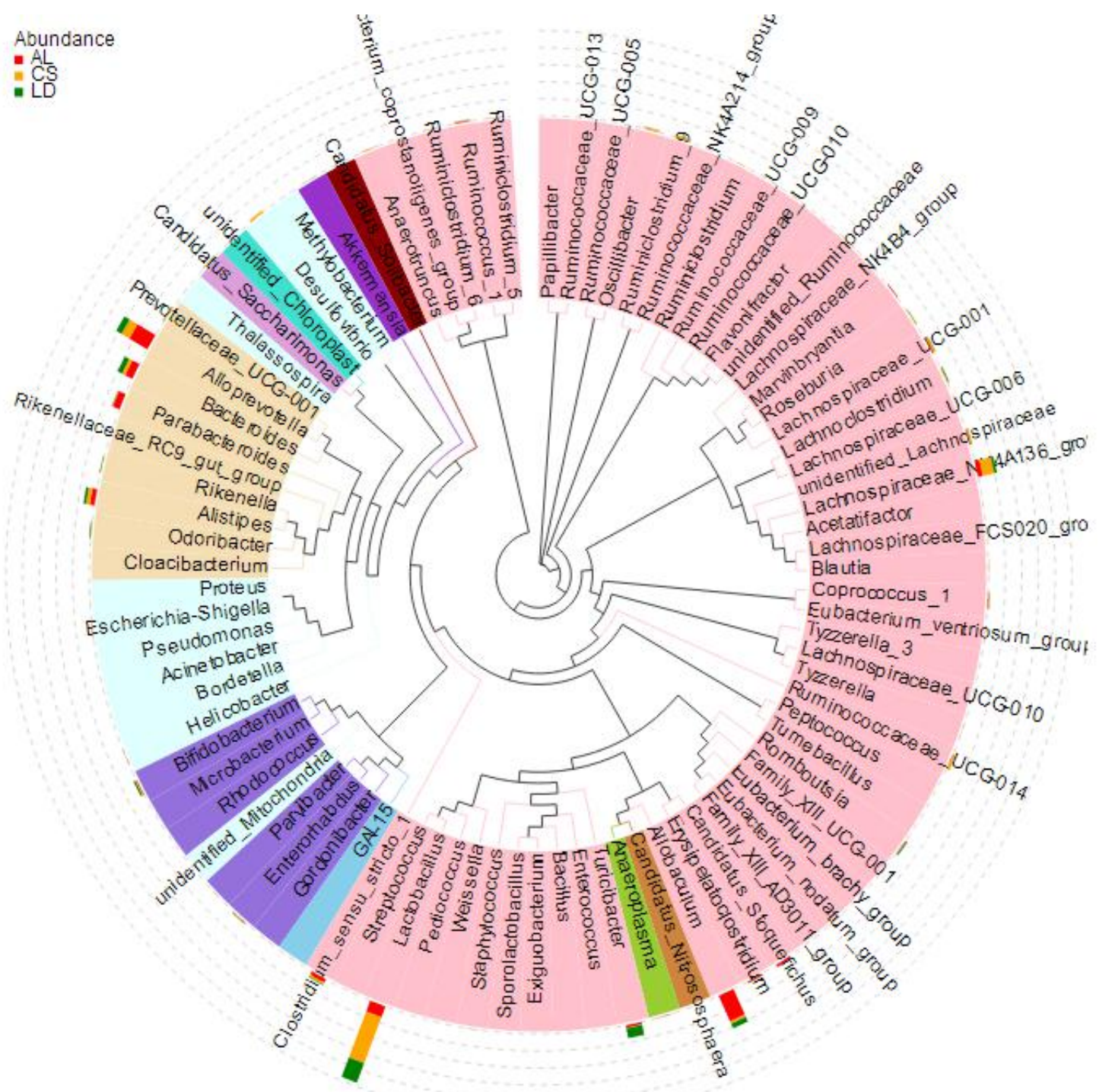




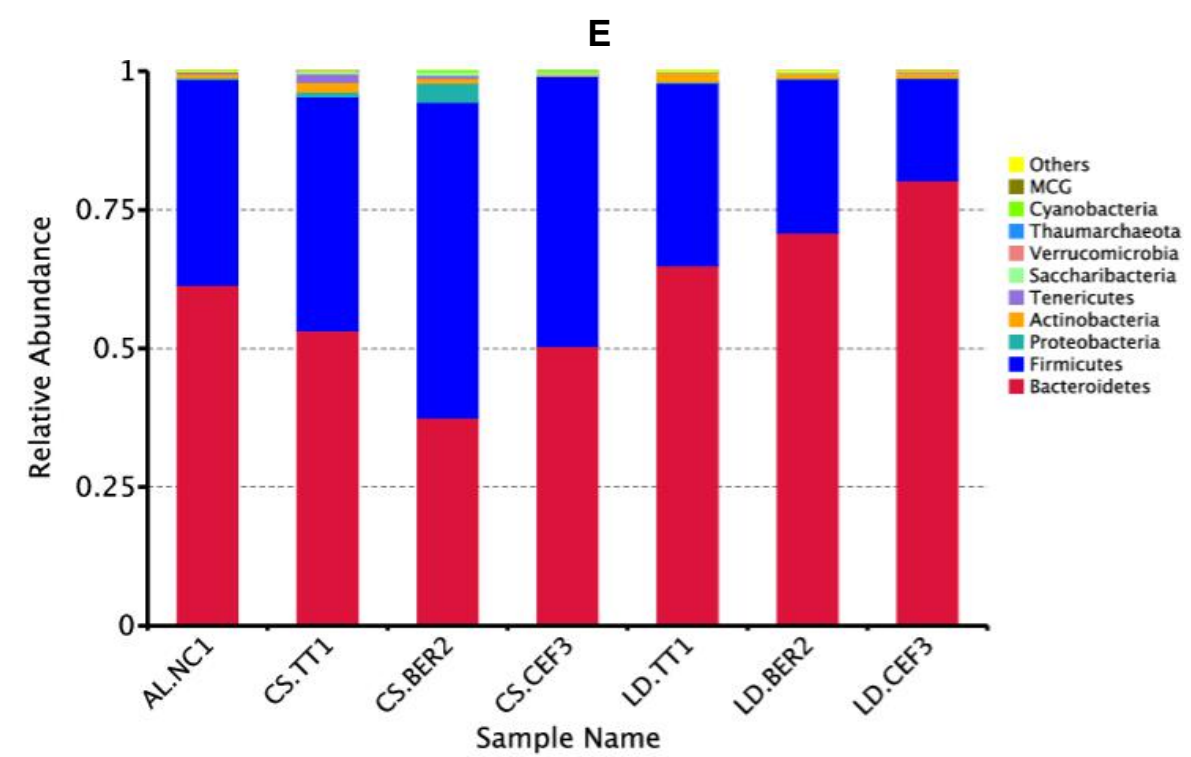

$\mathbf{F}$
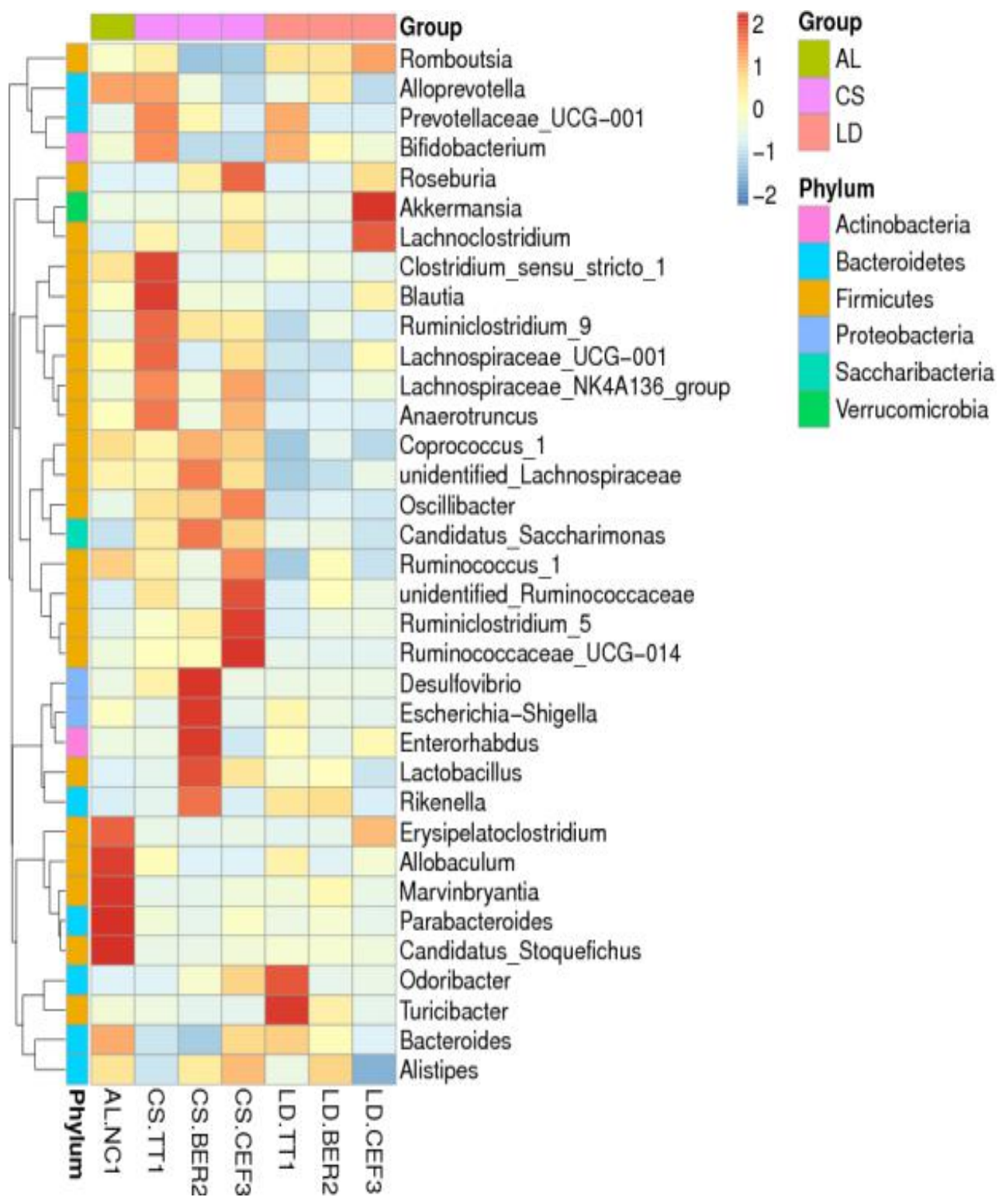

unidentified_Lachnospiraceae

Oscillibacter

Candidatus Saccharimonas

Ruminococcus_1

unidentified Ruminococcaceae

Ruminococcaceae_UCG-014

Desulfovibrio

Enterorhabdus

Lactobacillus

Erysipelatoclostridium

Allobaculum

Marvinbryantia

Candidatus Stoquefichus

Odoribacter

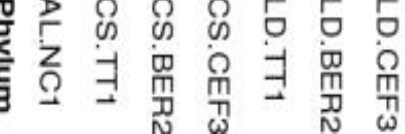



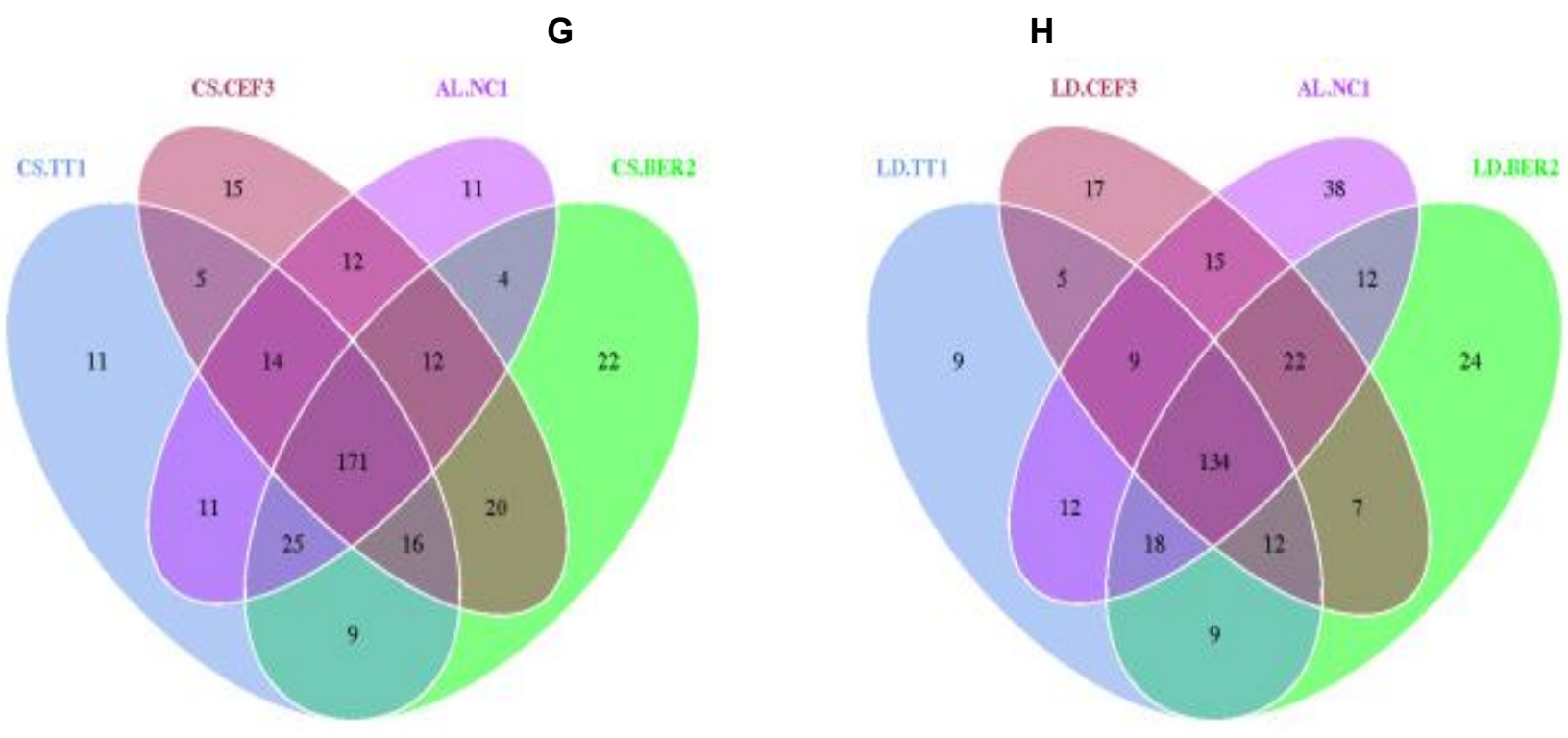

I

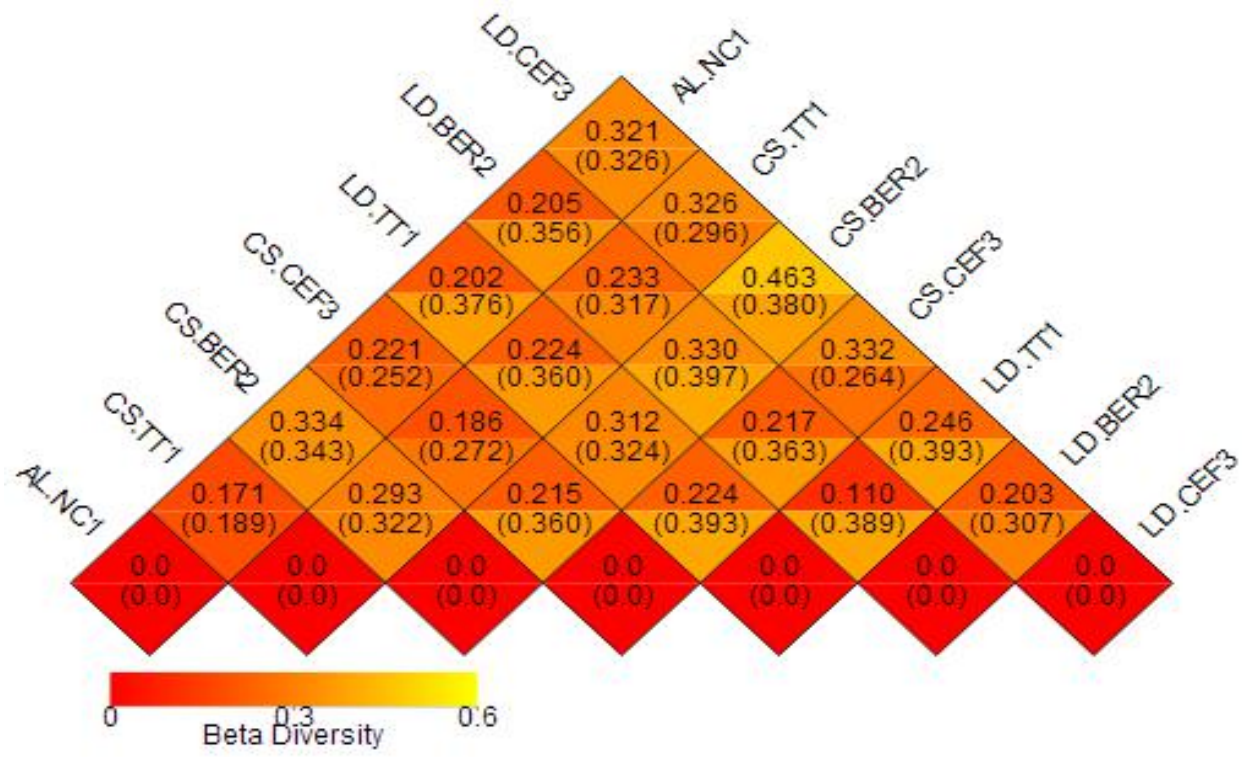

Figure 1. The gut microbiota profiles in AL, CS, LD, BER, and CEF mice. (A) The relative abundance of Top 10 genera in AL, CS, and LD mice. (B) Top 35 abundant genera in AL, CS, and LD mice. (C) Top 100 OTUs in AL, CS, and LD mice. (D) Top 100 abundant genera in AL, CS, and LD mice. (E) The relative abundance of Top 10 genera in AL.NS1, CS.TT1, CS.BER2, CS.CEF3, LD.TT1, LD.BER2, and LD.CEF3. (F) Top 35 abundant genera in AL.NS1, CS.TT1, CS.BER2, CS.CEF3, LD.TT1, LD.BER2, and LD.CEF3. (G) Top 100 OTUs in AL.NS1, CS.TT1, CS.BER2, and CS.CEF3. (H) Top 100 OTUs in AL.NS1, LD.TT1, LD.BER2, and LD.CEF3. (I) $\beta$-diversity in AL.NS1, CS.TT1, CS.BER2, CS.CEF3, LD.TT1, LD.BER2, and LD.CEF3.

As a species belonging to mucin-degrading bacteria (MDB), A. muciniphila was not observed to be induced by CS because its abundance in CS.TT1 is the same as that in AL.NC1 (0.01\%). Surprisingly, CEF increases its abundance from $0.01 \%$ in CS.TT1 to $0.09 \%$ in CS.CEF3 as well as from 0 in LD.TT1 to $0.3 \%$ in LD.CEF. In contrast, BER can eradicate $A$. muciniphila (Table 1). These results highlighted $A$. muciniphila might only "eat" 
mucin but not "eat" CS, which means $A$. muciniphila is unlikely a CS-induced species of SSB. However, A. muciniphila might be survived and even flourished by antibiotics, such as CEF, via killing other bacteria.

Besides, BER also increases an abundance of the pathogen Escherichia-shigella from $0.10 \%$ in AL.NC1 to $0.70 \%$ in CS.BER2 or $0.20 \%$ in LD.TT1, but decreases its abundance to $0.001 \%$ in CS.TT1, $0.03 \%$ in LD.BER2 or 0 in LD.CEF3. Intriguingly, BER extremely increases the abundance of Lactobacillus, one genus of probiotic bacteria, but eradicate Bifidobacterium, another genus of probiotic bacteria (Table 1).

Table 1. The proportion of selective bacterial phyla and genera in AL, CS, LD, BER, and CEF mice.

\begin{tabular}{lrrrrrrr}
\multicolumn{1}{c}{ Phyla/Genera } & AL.NC1 & CS.TT1 & CS.BER2 & CS.CEF3 & LD.TT1 & LD.BER2 & LD.CEF3 \\
\hline Phylum:Proteobacteria & 0.30 & 0.80 & 3.00 & 0.007 & 0.20 & 0.09 & 0.04 \\
Genus: Desulfovibrio & 0.006 & 0.70 & 3.00 & 0.003 & 0 & 0 & 0 \\
Genus: Escherichia-shigella & 0.10 & 0.009 & 0.70 & 0 & 0.20 & 0.03 & 0 \\
Phylum:Verrucomicrobia & NT & NT & NT & NT & NT & NT & NT \\
Species:Akkermansia muciniphila & 0.01 & 0.01 & 0.00 & 0.09 & 0.00 & 0.00 & 0.30 \\
Phylum:Bacteroidetes & 61.00 & 53.00 & 38.00 & 50.00 & 65.00 & 71.00 & 80.00 \\
Genus: Rikenella & 0.001 & 0.05 & 0.70 & 0 & 0.40 & 0.40 & 0 \\
Phylum:Firmicutes & 37.00 & 42.00 & 57.00 & 49.00 & 33.00 & 28.00 & 18.00 \\
Genus: Lactobacillus & 6.00 & 8.00 & 43.00 & 24.00 & 13.00 & 16.00 & 3.00 \\
Phylum: Actinobacteria & 0.80 & 2.00 & 0.90 & 0.10 & 2.00 & 0.90 & 0.80 \\
Genus: Bifidobacterium & 0.50 & 2.00 & 0.00 & 0.003 & 1.00 & 0.70 & 0.40 \\
\hline Note: The proportion is & & &
\end{tabular}

Note: The proportion is represented by the percentage (\%). NT: not tested.

Although an interaction of the gut microbiota is complex, it was still aware that Desulfovibrio and Rikenella are positively or dependently correlated, whereas Rikenella and Akkermansia are negatively or independently correlated (Figure 2E). It should be understandable because Rikenella as SSB provide sulfate to Desulfovibrio as SRB, but both Rikenella and Akkermansia are SSB.

CS exhibits a growth inhibitory effect on Bacteroidetes by decreasing its abundance from $61 \%$ to $53 \%$, including decreasing the abundance of Bacteroides from $4 \%$ to $1 \%$. While CEF eradicates the pathogen Escherichia-shigella and also decreases the abundance of the probiotic Bifidobacterium, BER cannot eradicate Escherichia-shigella, but can kill Bifidobacterium. It was evident that Desulfovibrio use sulfate to generate hydrogen sulfide, which can be convert to thiosulfate and further oxidized to tetrathionate, thereby promoting the overgrowth of Y-proteobacteria, including Escherichia-shigella (Rajilić-Stojanović et al. 2015). Briefly, CS enriches Desulfovibrio and Rikenella, by which a metabolic chain that links O-glycan sulfate induction to sulfatase secretion, sulfate release and sulfate reduction is formed. BER potentiates such an association by further enriching Desulfovibrio to $3 \%$ and Rikenella to $2 \%$. Additionally, A. muciniphila is enriched by CEF but not by CS and LD.

It was previously confirmed that SRB are colonized in the human gut accounting for a proportion of about $50 \%$ among the commensal microbes (ljssennager et al., 2015), including $66 \%$ of Desulfovibrio and $16 \%$ of Desulfobulbus (Leschelle et al., 2005). Although an almost equal proportion of SRB might be anticipated in this study, we only identified 
bioRxiv preprint doi: https://doi.org/10.1101/142588; this version posted May 27, 2017. The copyright holder for this preprint (which was not certified by peer review) is the author/funder, who has granted bioRxiv a license to display the preprint in perpetuity. It is made available under aCC-BY-NC-ND 4.0 International license.

Desulfovibrio in AL.TT1 and CS.TT1 for $0.01 \%$ and $0.70 \%$, suggesting a potential existence of SRB in the unidentified SSB (Rey et al., 2013). SRB exploit sulfate as a terminal electron acceptor in their respiratory chains to oxidize the organic compounds and hydrogen into hydrogen sulfide, which can directly induce free radicals, prompt DNA damage, and increase the risk of colon cancer (Attene-Ramos et al., 2007). For example, $A$. muciniphila as SSB and SRB can reduce sulfate to sulfide (ljssennager et al., 2015).

A

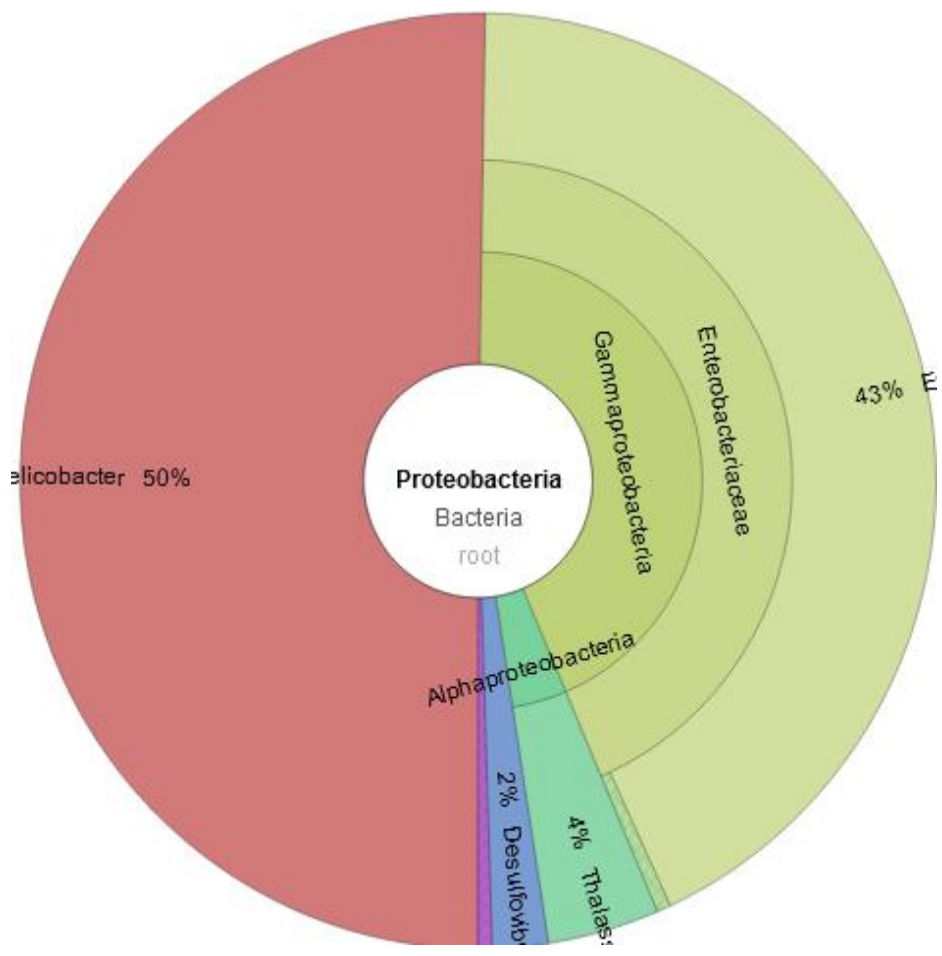

C

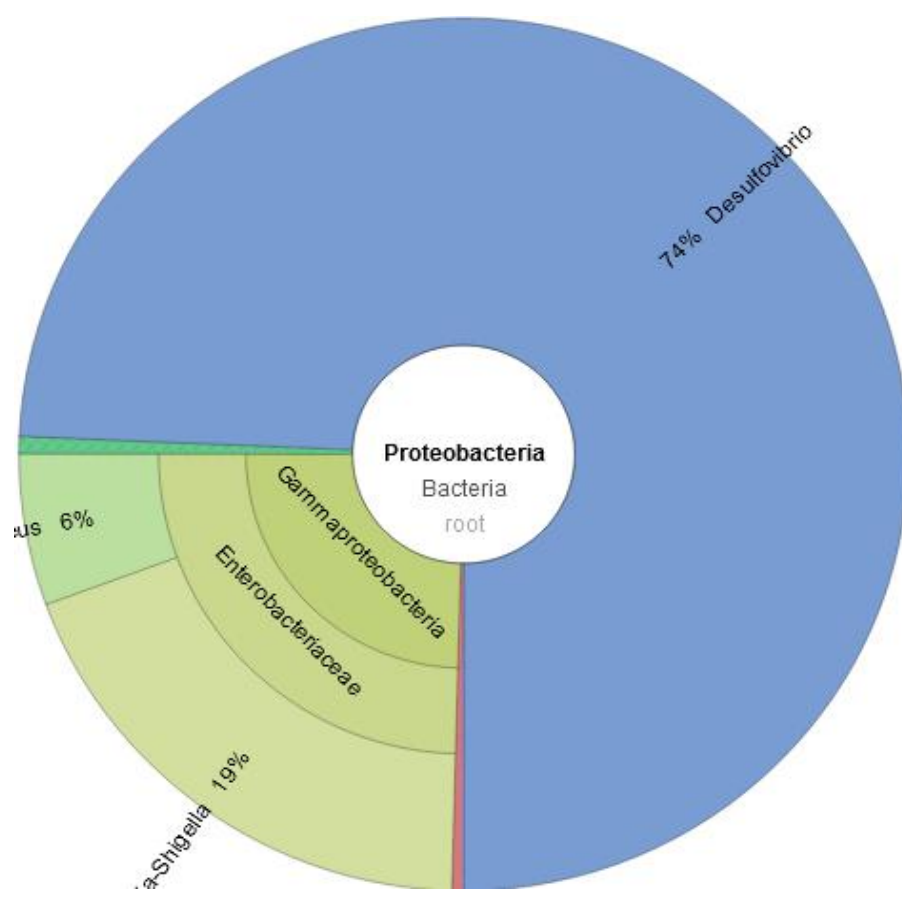

B

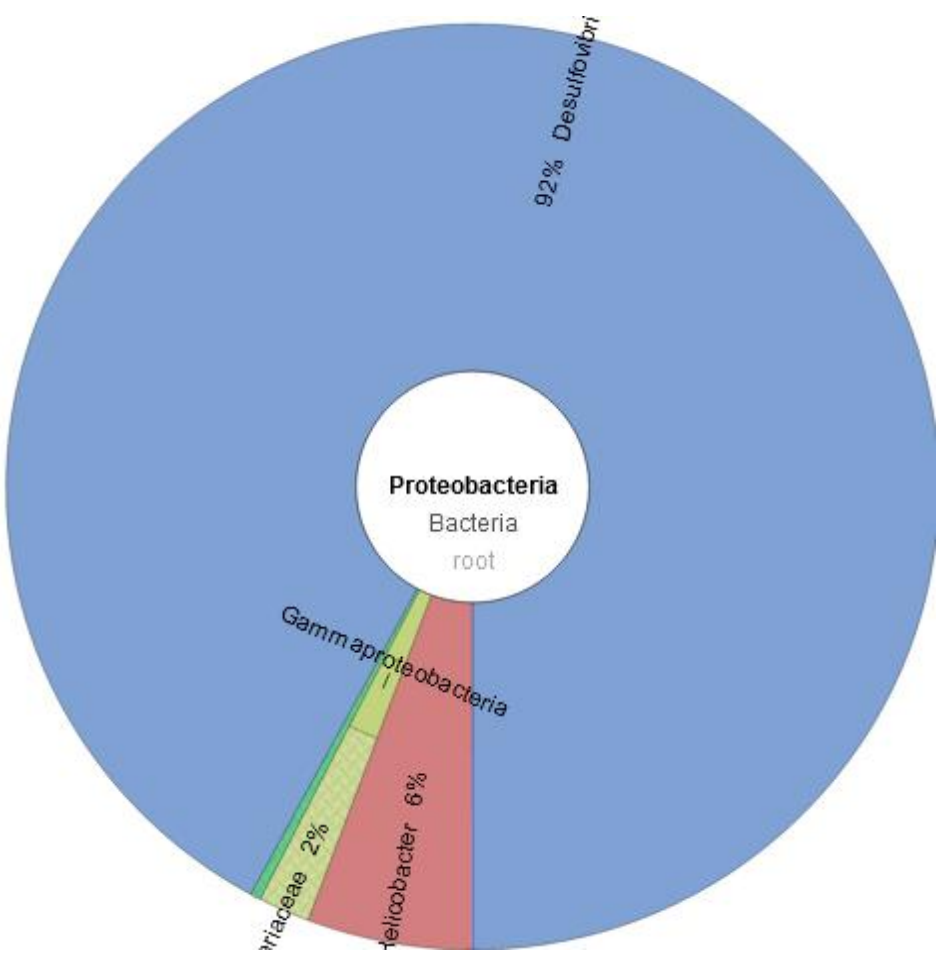

D

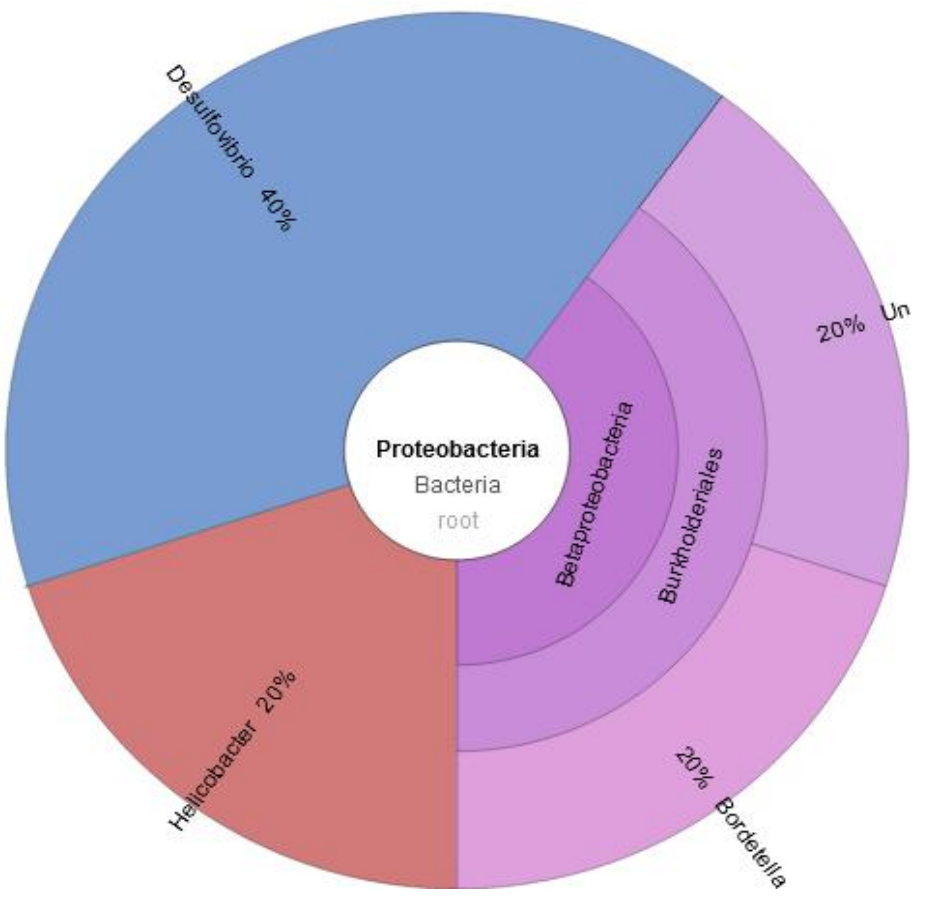


bioRxiv preprint doi: https://doi.org/101101/142588; this version posted May 27 2017. The copyright holder for this preprint (which was not certified by peer review) is the author/funder, who has granted bioRxiv a license to display the preprint in perpetuity. It is made available under aCC-BY-NC-ND 4.0 International license.

$\mathrm{E}$

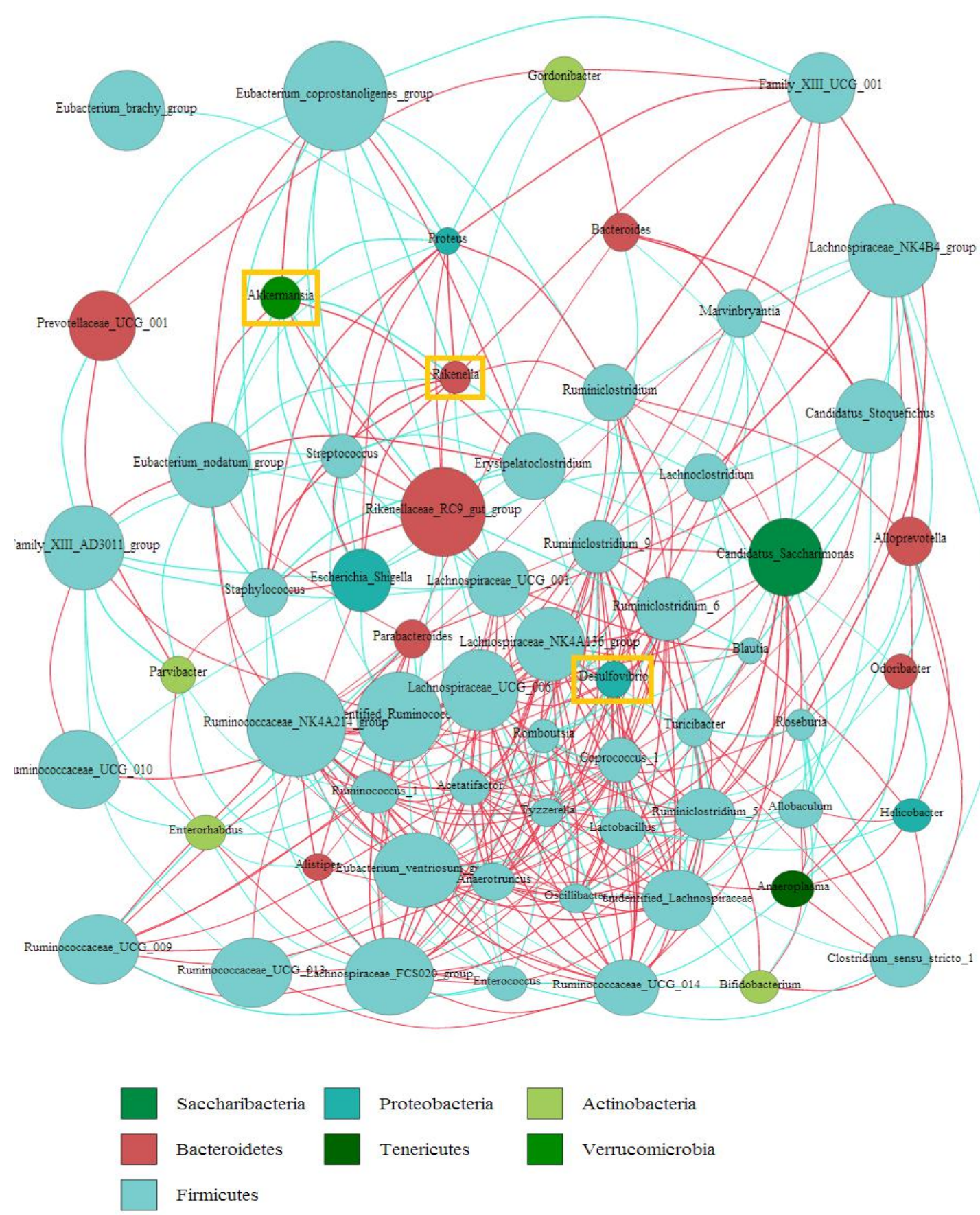

Figure 2. The abundance of Desulfovibrio in Proteobacteria and interactions among Desulfovibrio, Rikenella, and Akkermansia. (A) The percentage of Desulfovibrio in AL.NC1. (B) The percentage of Desulfovibrio in CS.TT1. (C) The percentage of Desulfovibrio in CS.BER2. (D) The percentage of Desulfovibrio in CS.CEF3. (E) An interactive network among Desulfovibrio, Rikenella, and Akkermansia (yellow boxes), in which the size of a dot represents an average relative abundance in a specific genus, and the line between every dot indicates a positive (red) or negative (blue) correlation.

Colon Lesions Are Dependent Upon Desulfovibrio And Rikenella But Colon Repairs Are Enhanced By A. muciniphila 
bioRxiv preprint doi: https://doi org/10.1101/142588; this version posted May 27, 2017. The copyright holder for this preprint (which was not certified by peer review) is the author/funder, who has granted bioRxiv a license to display the preprint in perpetuity. It is made available under aCC-BY-NC-ND 4.0 International license.

To assess a causal linkage of SSB/SRB to mucus layer integrity, we detected the secretary leukocyte protease inhibitor (SLPI), a hallmark of colon lesions (Clauss et al., 2002), in the descending colon lining of all tested mice. As compared with AL.NC1 (Figure 3A1), the color that correlates with the SLPI level was discernible to be dark brown in CS.TT1 (Figure 3A2) and darker brown in CS.BER (Figure 3A3), but lighter brown in CS.CEF (Figure 3A4). Among the LD group, their colon linings generally show a relatively lower SLPI level than the CS group, in which LD.CEF3 shows the lowest SLPI level (Figure 3A5-3A7).

Interestingly, the overexpression of SLPI is likely correlated with the abundance of Desulfovibrio and Rikenella (see Table 1). For example, CS.TT1 and CS.BER2 with abundant Desulfovibrio and Rikenella show the higher SLPI levels than AL.NC1. In contrast, CS.CEF3 with trace-number Desulfovibrio but without Rikenella shows the lower SLPI levels than CS.TT1 and CS.BER2. In similar, LD.TT1 and LD.BER2 with trace-number Rikenella but without Desulfovibrio also exhibit the lower SLPI levels. In particular, LD.CEF3 without Desulfovibrio and Rikenella show the lowest SLPI levels.

As compared with AL.NC1 (Figure 3B1), it was clearly that the pink dots representing the merged blue fluorescence of DNA and red fluorescence of mucin 1 (MUC1) are much less in the colon mucus linings of CS.BER2 than those of CS.TT1 and CS.CEF3, implying a dramatic downregulation of MUC1 in CS.BER2. At the same time, the colon mucus linings of CS.BER2 also exhibit many mesh-like structures with huge holes (Figure 3B2-3B4), also implying an extreme downregulation of MUC1 and other subtypes of mucins in CS.BER2. Interestingly, CS.BER2 does not harbor A. muciniphila, whereas AL.NC1, CS.TT1 and CS.CEF3 carry $A$. muciniphila (see Table 1), suggesting a possible correlation of MUC1 overexpression and colon repairs with $A$. muciniphila presence.

In contrast, it was unambiguously distinguished that LD.CEF3 possesses much more pink dots and much less mesh-like structures with huge holes than LD.TT1 and LD.BER2 (Figure 3B5-3B7), indicating an overexpression of MUC1 in LD.CEF3. Coincidentally, LD.CEF3 harbors abundant $A$. muciniphila, whereas LD.TT1 and LD.BER2 does not carry A. muciniphila (see Table 1).

A1

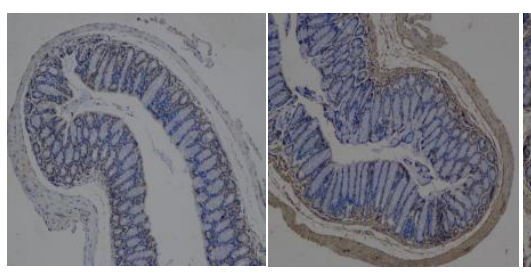

B1

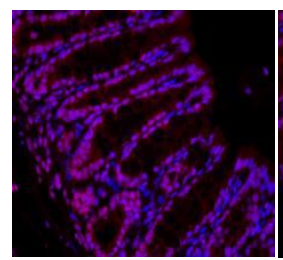

A3

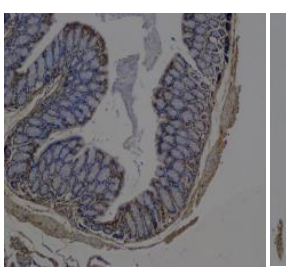

B3
A4

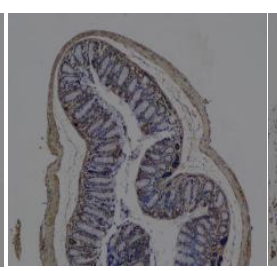

B4
A5

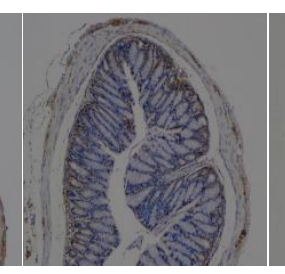

B5
A6

A7

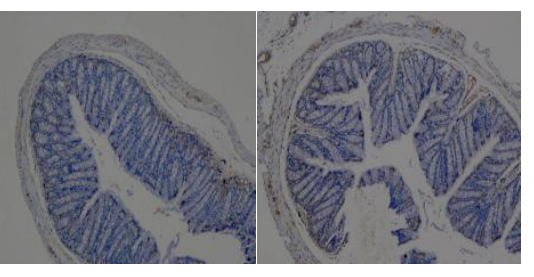

B6

B7
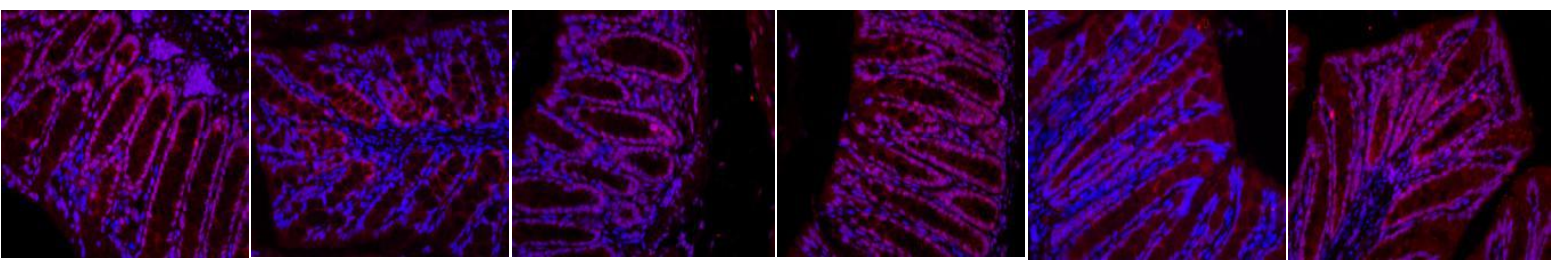

Figure 3. Expression of SLPI and MUC1 in the colon linings of AL, CS, LD, BER, and CEF mice. (A) SLPI staining (× 200). (B) MUC1 fluorescence (× 400). A1-A7 and B1-B7 represent AL.NC1, CS.TT1, 
CS.BER2, CS.CEF3, LD.TT1, LD.BER2, and LD.CEF3, respectively. SLPI was stained into the brown color around the colon linings. The red fluorescence represents MUC1, and the blue fluorescence represents DNA stained by DPCI.

The gut bacteria MDB/SSB are classified into two categories: one is mucin specialists that only grow on mucin O-glycans as a sole polysaccharide source (such as $A$. muciniphila and Barnesiella intestinihominis), and another is mucin generalists that grow on several other polysaccharides (such as $B$. thetaiotaomicron and Bacteroides caccae) (Desai et al., 2016). Using the gut microbiota metagenomic analysis, we identified $A$. muciniphila and Rikenella. Nevertheless, it should be predicted that some other MDB/SSB might be included in the unidentified Bacteroidetes, which exist in LD mice for $65 \%$ and in CS mice up to $53 \%$.

\section{Endotoxin Leakage Leads To Prompted LPS Circulation and Accelerated Anti-LPS Induction}

Because of colon mucus openings, LPS derived from Gram negative bacteria can be readily leaked into the blood stream and induce anti-LPS induction. To evaluate the interactive changes of LPS and anti-LPS, we measured at first their peripheral (serum and muscular) levels. As shown in Figure 4A-4B, it was unexpectedly observed that the serum and muscular LPS levels are almost declined among all treated mice, even lower than AL mice, suggesting CS/LD might elevate LPS levels at first, then induce anti-LPS to neutralize LPS, and eventually decline LPS levels. Subsequently, we determined the visceral (adipose, hepatic, cardiac and cerebral) LPS levels (Figure 4C-4F). It was seen that the multiple organ LPS levels in treated mice are also nearly equal to or lower than those in AL mice, also suggesting a trend of an elevated LPS level at first and then a declined LPS level in CS/LD mice.

As evidence supporting the above prediction that LPS induces anti-LPS at first, anti-LPS secondly neutralizes LPS, and eventually declines the LPS levels, it was obviously that the multiple organ anti-LPS levels in all treated mice are generally equal to or higher than those in AL mice. Furthermore, it was clearly that antibiotic use, of course, affects the dynamic interaction between LPS and anti-LPS as antibiotics might decline or elevate the LPS levels. The muscular, adipose, cardiac, and cerebral anti-LPS levels (Figure 4G-4J) in CS.BER2 are higher than AL.NC1. These results indicated an active immunostimulatory response is triggered in CS.BER2 to give rise to an adverse inflammatory outcome.

A

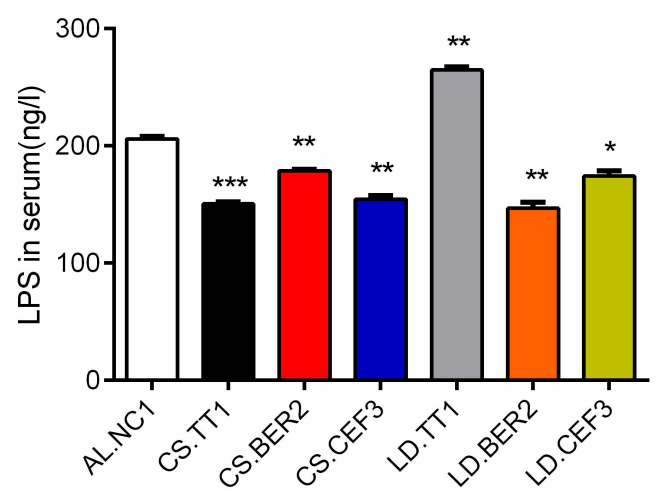

B

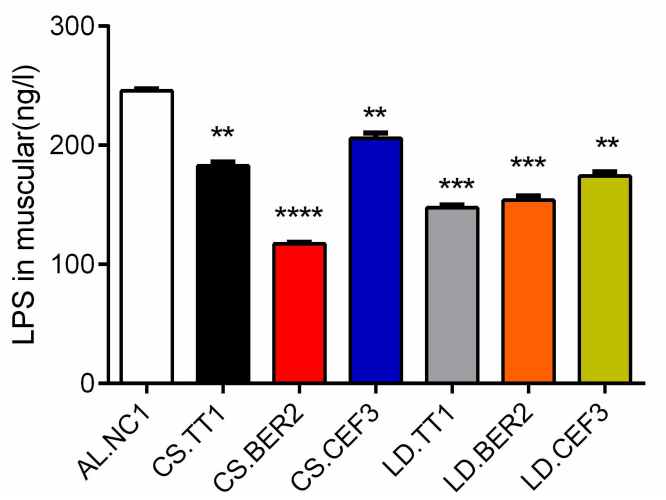


C

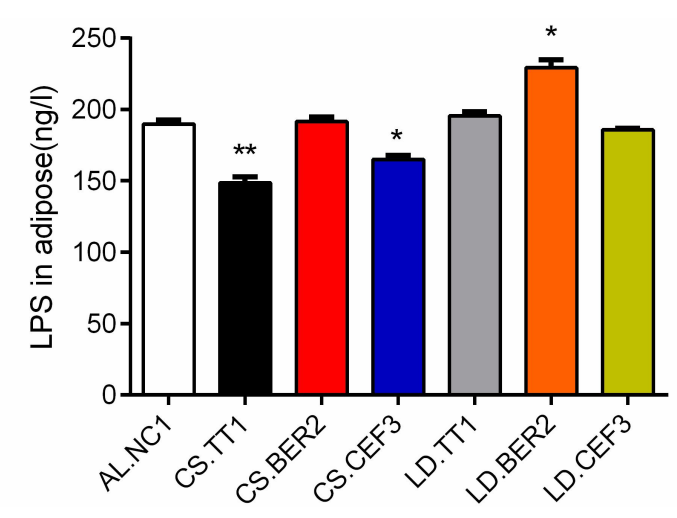

E

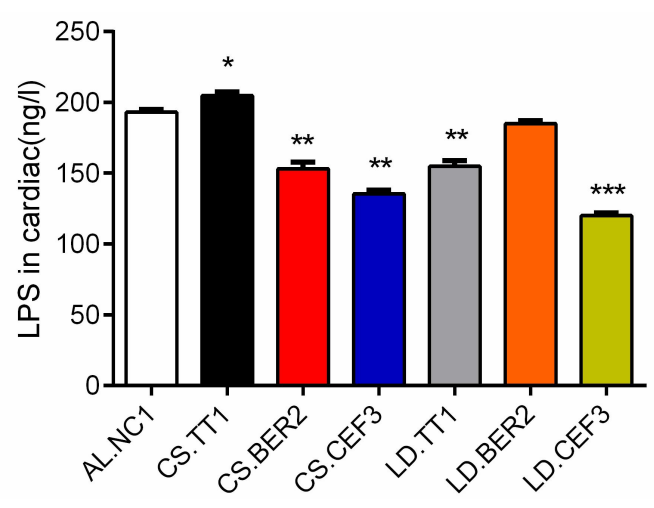

G

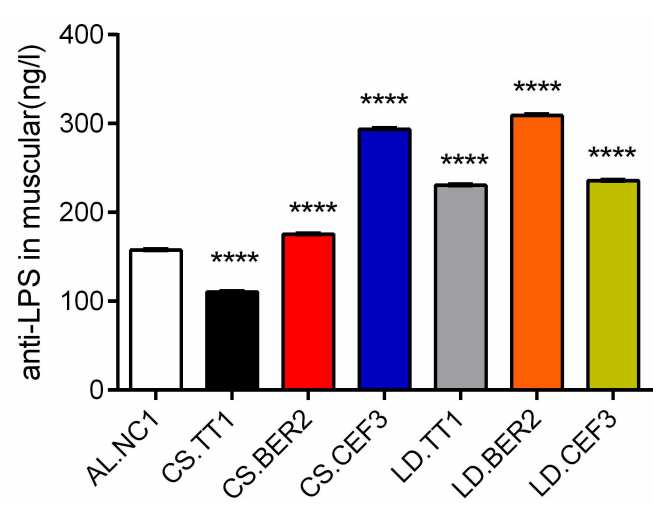

I

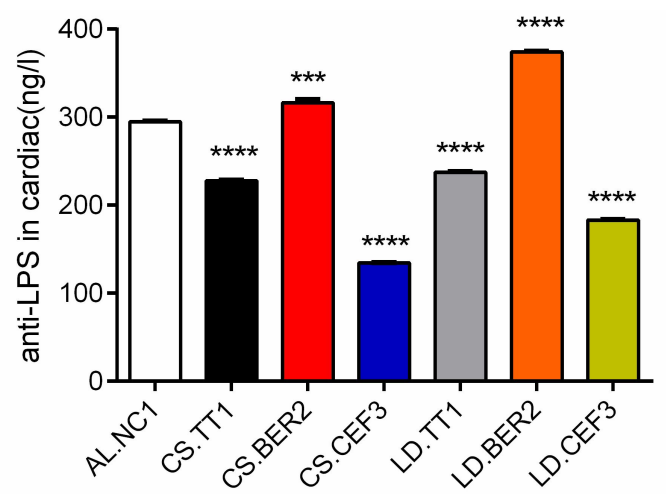

D

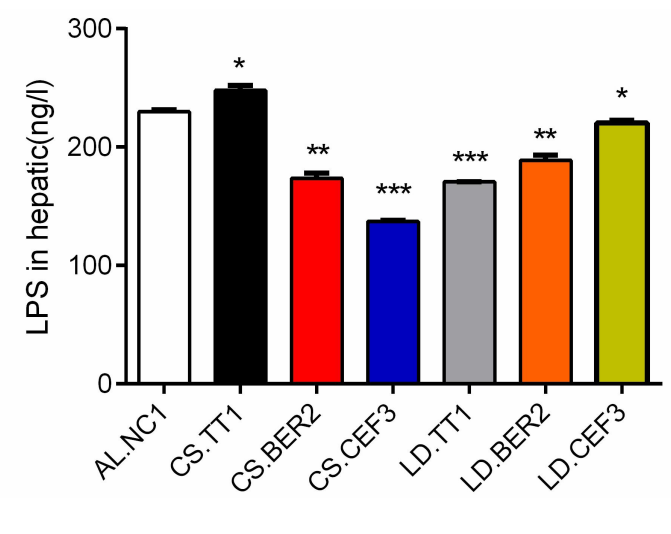

$\mathbf{F}$

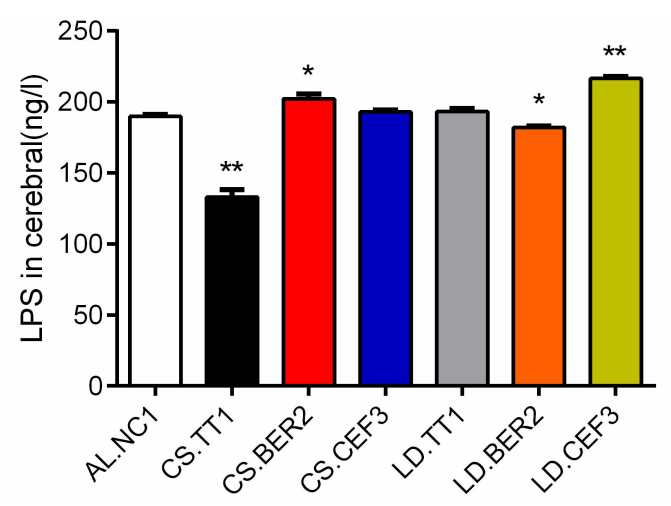

H

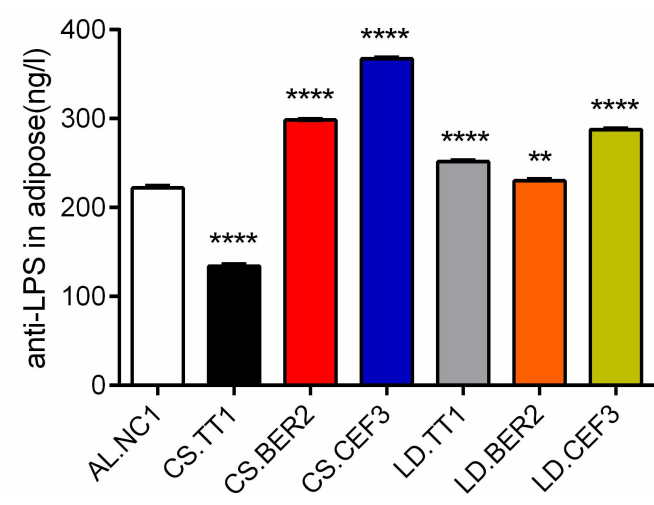

J

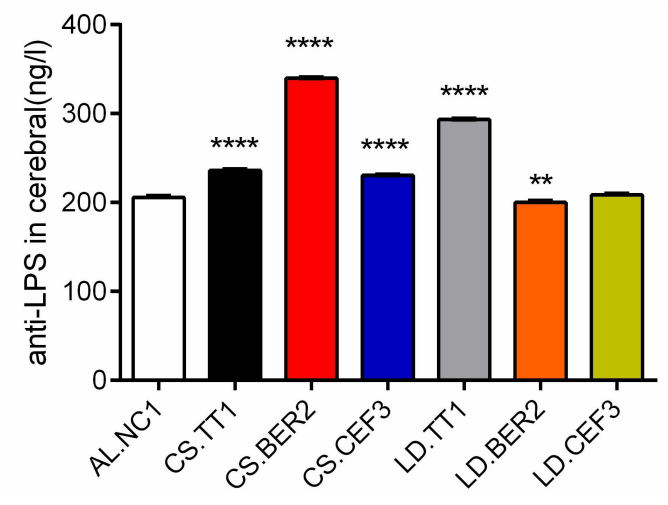


bioRxiv preprint doi: https://doi.org/10.1101/142588; this version posted May 27, 2017. The copyright holder for this preprint (which was not certified by peer review) is the author/funder, who has granted bioRxiv a license to display the preprint in perpetuity. It is made available under aCC-BY-NC-ND 4.0 International license.

Figure 4. The serum and muscular levels of LPS, anti-LPS and TNF-a in AL, CS, LD, BER, and CEF mice. (A) LPS levels in the serum. (B) LPS levels in the muscular tissue. (C) LPS levels in the adipose tissue. (D) LPS levels in the hepatic tissue. (E) LPS levels in the cardiac tissue. (F) LPS levels in the cerebral tissue. (G) Anti-LPS levels in the muscular tissue. (H) Anti-LPS levels in the adipose tissue. (I) Anti-LPS levels in the cardiac tissue. (J) Anti-LPS levels in the cerebral tissue.

\section{Systemic Inflammation Is Followed By Upregulated Mitochondrial Biomarkers}

To monitor whether LPS systemically induces the pro-inflammatory responses, we measured the peripheral levels of tumor necrosis factor a (TNF-a), a typical and major pro-inflammatory cytokine. Consequently, TNF-a maintains a relatively low level in the serum among almost all groups of mice except for LD.TT1, but a high level in the muscular tissue in CS.TT1, CS.CEF3 and LD.BER2 (Figure 5A and 5B). Among the visceral tissues, while the cardiac tissue shows the highest TNF-a levels, the cerebral tissue exhibits the lowest TNF-a levels (Figure 5C-5F). Upon treatment by antibiotics, the TNF-a levels are either elevated or declined, implying a complicated interaction among the gut microbiota communities, antibiotics and immune responses. Accordingly, JAK2 and STAT3 that convey an inflammatory signal and initiate cytokine transcription are generally upregulated in the hepatic tissues of CS and LD mice with or without antibiotic supplementation, thereby suggesting an enhanced pro-inflammatory response (Figure 5G and Table 2).

To establish an association of pro-inflammation with mitochondrial biogenesis, we quantified some critical proteins responsible for mitochondrial biogenesis and mitochondrial marker proteins in all tested mice. It was clarified that AMPK and SIRT1 transducing an energy deprival signal are globally upregulated in the hepatic and muscular tissues of CS and LD mice with or without antibiotic supplementation although the hepatic tissues exhibit the higher levels than the muscular tissues (Figure 5G and Table 2). Accordingly, MFN2 as a mitochondrial biomarker indicating mitochondrial turnover and UCP1 as an oxidative phosphorylation uncoupling indicator reflecting a conversion from energy deposition to energy expenditure are typically upregulated in the hepatic and muscular tissues of CS and LD mice with or without antibiotic supplementation although give rise to the higher levels in the muscular tissue than those in the hepatic tissue (Figure $5 \mathrm{G}$ and Table 2). These results indicated a pro-inflammatory response would induce mitochondrial biogenesis to compensate a functional deficiency of the damaged mitochondria.

A

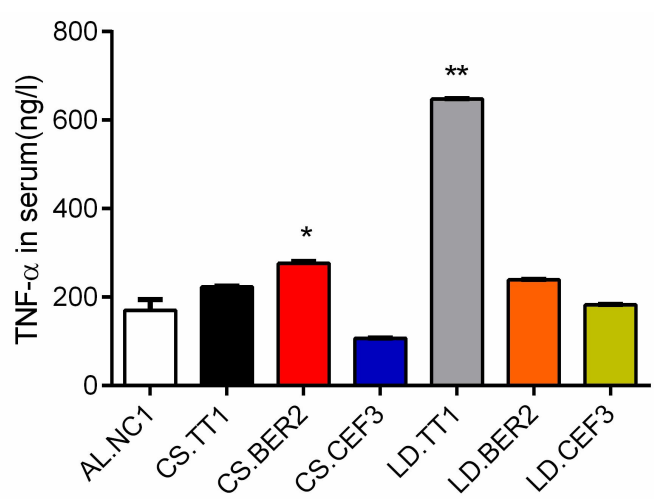

B

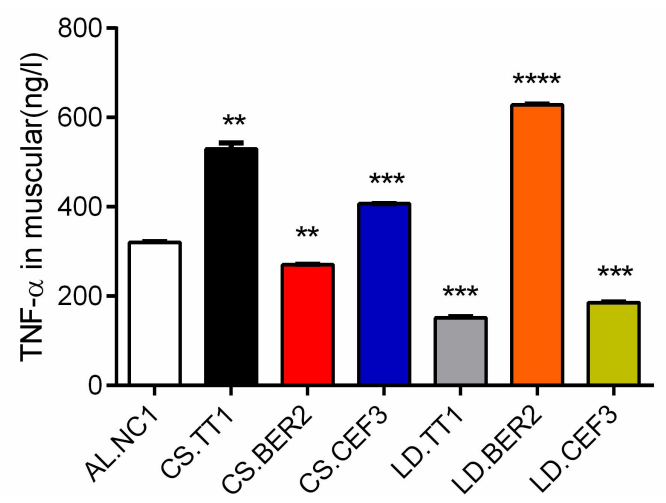


C

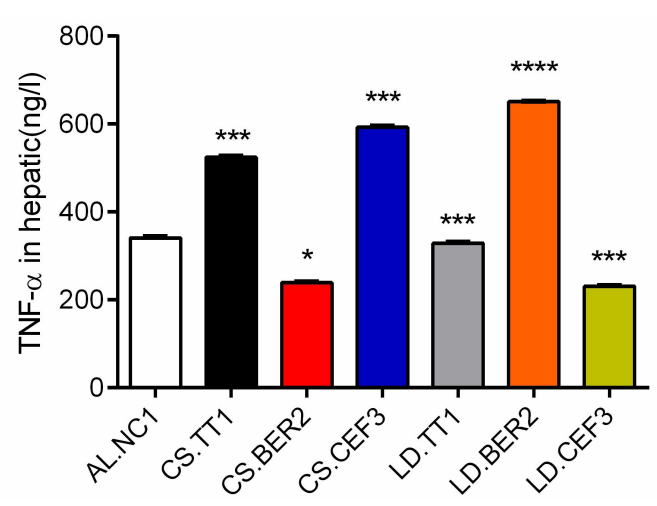

E

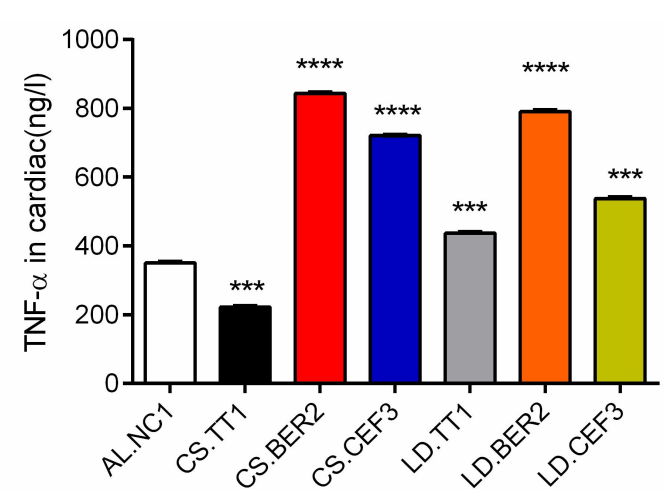

D

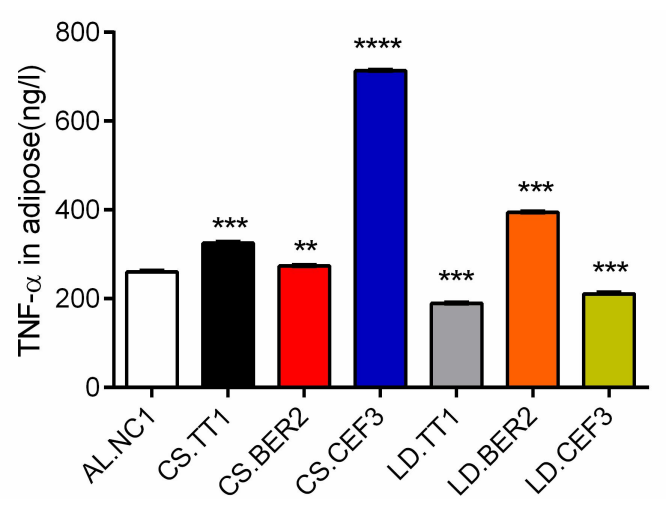

$\mathbf{F}$

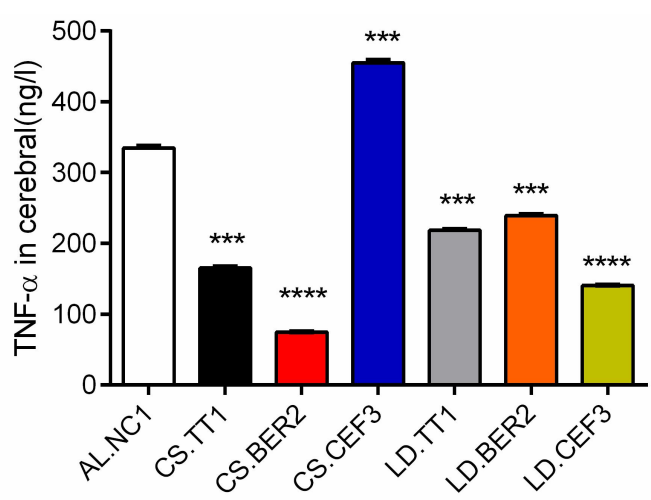

G

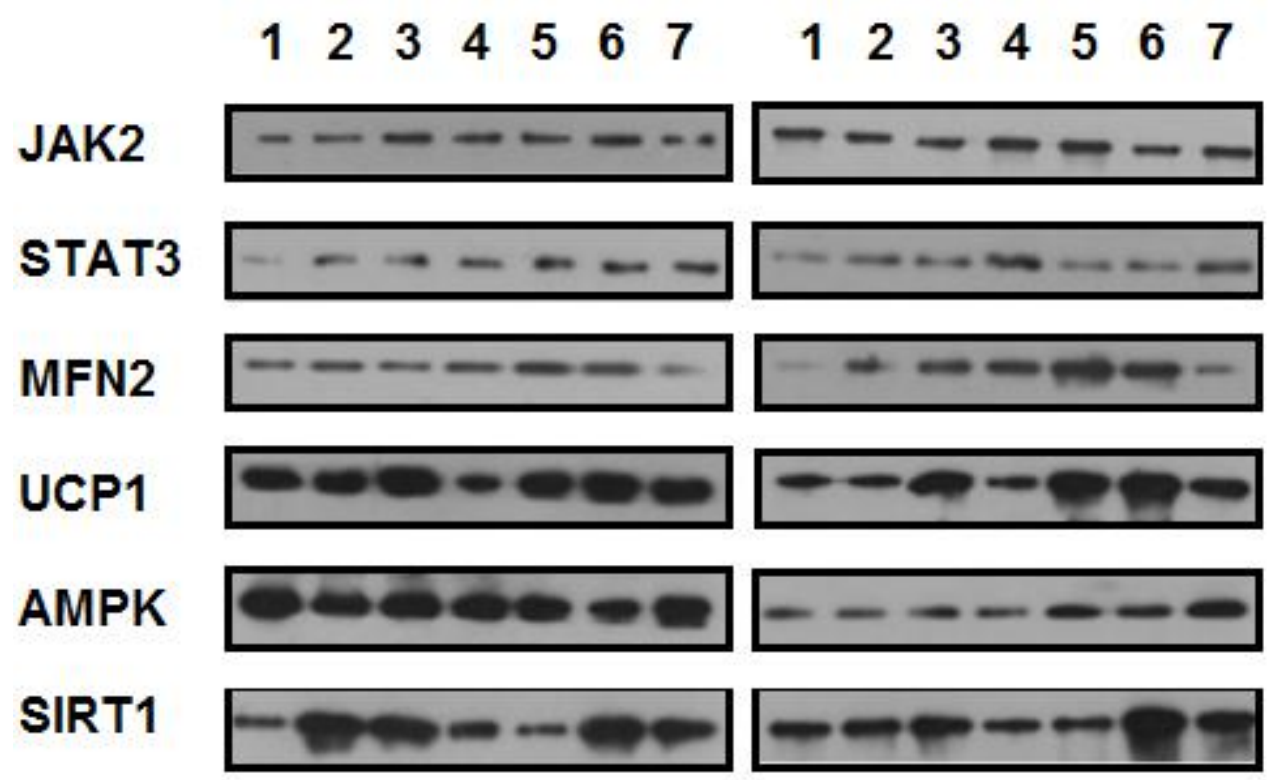

Figure 5. The profiling of pro-inflammatory responses and mitochondrial biogenesis in AL, CS, LD, BER, and CEF mice. (A) TNF-a levels in the serum. (B) TNF-a levels in the muscular tissue. (C) TNF-a levels in the hepatic tissue. (D) TNF-a levels in the adipose tissue. (E) TNF-a levels in the cardiac tissue. (F) TNF-a levels in the cerebral tissue. (G) The hepatic levels (left panel) and muscular levels (right panel) of some critical proteins responsible for mitochondrial biogenesis and mitochondrial biomarker proteins in all tested mice, in which No.1-7 represent AL.NC1, CS.TT1, CS.BER2, CS.CEF3, LD.TT1, LD.BER2, and 
LD.CEF3, respectively. MFN2: mitofusin 2. UCP1: uncoupling protein 1. AMPK: adenosine

5'-monophosphate-activated protein kinase. JAK2: Janus kinase 2. STAT3: signal transducer and activator of transcription 1 protein. SIRT1: silent mating type information regulation 2 homolog (sirtuin) type 1. GAPDH: glyceraldehyde-3-phosphate dehydrogenase.

Table 2 The relative gray scale values of target proteins in the hepatic or muscular tissue of AL, CS, LD, BER, and CEF mice.

\begin{tabular}{lccccccc} 
& AL.NC1 & CS.TT1 & CS.BER2 & CS.CEF3 & LD.TT1 & LD.BER2 & LD.CEF3 \\
\hline Hepatic & & & & & & & \\
JAK2/GAPDH & 0.2469 & 0.3171 & 0.3614 & 0.3580 & 0.3293 & 0.4878 & 0.2561 \\
STAT3/GAPDH & 0.1235 & 0.1829 & 0.2169 & 0.2593 & 0.2927 & 0.3171 & 0.3537 \\
AMPK/GAPDH & 1.5802 & 1.1220 & 1.1807 & 1.1728 & 1.0584 & 0.9390 & 1.4756 \\
SIRT1/GAPDH & 0.4198 & 1.0732 & 1.0482 & 0.6173 & 0.3415 & 1.1951 & 0.8293 \\
MFN2/GAPDH & 0.3210 & 0.3902 & 0.3614 & 0.4691 & 0.5854 & 0.5976 & 0.1829 \\
UCP1/GAPDH & 1.1111 & 1.1098 & 1.4458 & 0.6173 & 1.1585 & 1.5122 & 1.2195 \\
\hline Muscular & & & & & & & 0.975 \\
JAK2/GAPDH & 1.0000 & 0.7500 & 0.7179 & 0.9744 & 0.9487 & 0.7750 & 0.9744 \\
STAT3/GAPDH & 0.2564 & 0.3750 & 0.4103 & 0.5385 & 0.3590 & 0.3750 & 0.4615 \\
AMPK/GAPDH & 0.7179 & 0.6750 & 0.7692 & 0.6923 & 1.0513 & 0.9500 & 1.4872 \\
SIRT1/GAPDH & 1.1282 & 1.2250 & 1.3333 & 0.7692 & 0.7436 & 2.4500 & 2.0513 \\
MFN2/GAPDH & 0.2564 & 0.4500 & 1.0256 & 1.0513 & 1.4103 & 1.2750 & 0.3846 \\
UCP1/GAPDH & 0.9744 & 0.7500 & 2.0513 & 0.7692 & 2.3077 & 2.3500 & 1.4615 \\
\hline
\end{tabular}

Note: The ratios were calculated from the gray scale value of a target protein dividing that of GAPDH.

\section{Disordered And Dysfunctional Mitochondria Are Correlated With Augmented} Glycolytic product Accumulation And Attenuated Energetic Compound Production Due to an inflammation-induced hypoxic niche with insufficient oxygen supply, the nascent mitochondria might be dysfunctional, i.e., electron transport along the respiratory chain is interrupted. Under such a condition, mitophagy might be initiated and dysfunctional mitochondria depleted, eventually leading to a net decrease in mitochondrial copy numbers. From the electron microphotographs, more mitochondria were observed in the adipose tissues of AL.NC1 (Figure 6A1) than those in the adipose tissue of other mice (Figure 6A2-6A7). Similarly, more mitochondria were also seen in the muscular tissues of AL.NC1 (Figure 6B1) than those in other mice (Figure 6B2-6B7).

Accordingly, it was predicted that insufficient or dysfunctional mitochondria should compromise the anaerobic glucose degradation, leading to an elevated lactic acid (LA) level and a declined adenosine triphosphate (ATP) level. As depicted in Figure 6C, the muscular LA levels are globally elevated in all tested mice, but the hepatic LA levels are almost equal to or mildly higher than AL.NC1 (Figure 6D), reflecting a differential efficiency of the functional recovery of mitochondria. Accordingly, a higher muscular ATP level was detected in AL.NC1 compared to other treated mice (Figure 6E), whereas the lowest hepatic ATP level was determined in CS.BER2 among all treatment groups of mice (Figure $6 F)$. These results unraveled a clear correlation of mitochondrial dysfunction with high-level LA and low-level ATP, in which the highest LA and lowest ATP levels in the hepatic tissues 
bioRxiv preprint doi: https://doi org/10.1101/142588; this version posted May 27, 2017. The copyright holder for this preprint (which was

A

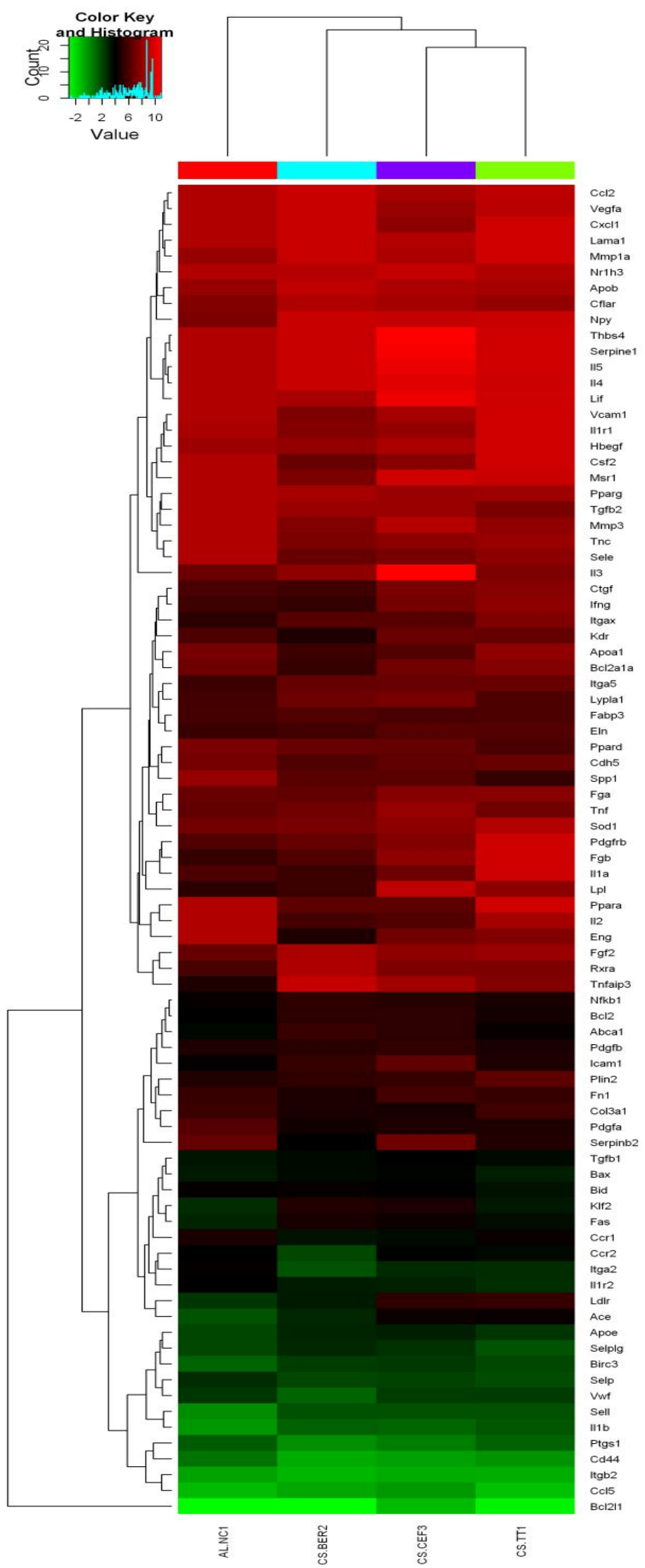

B

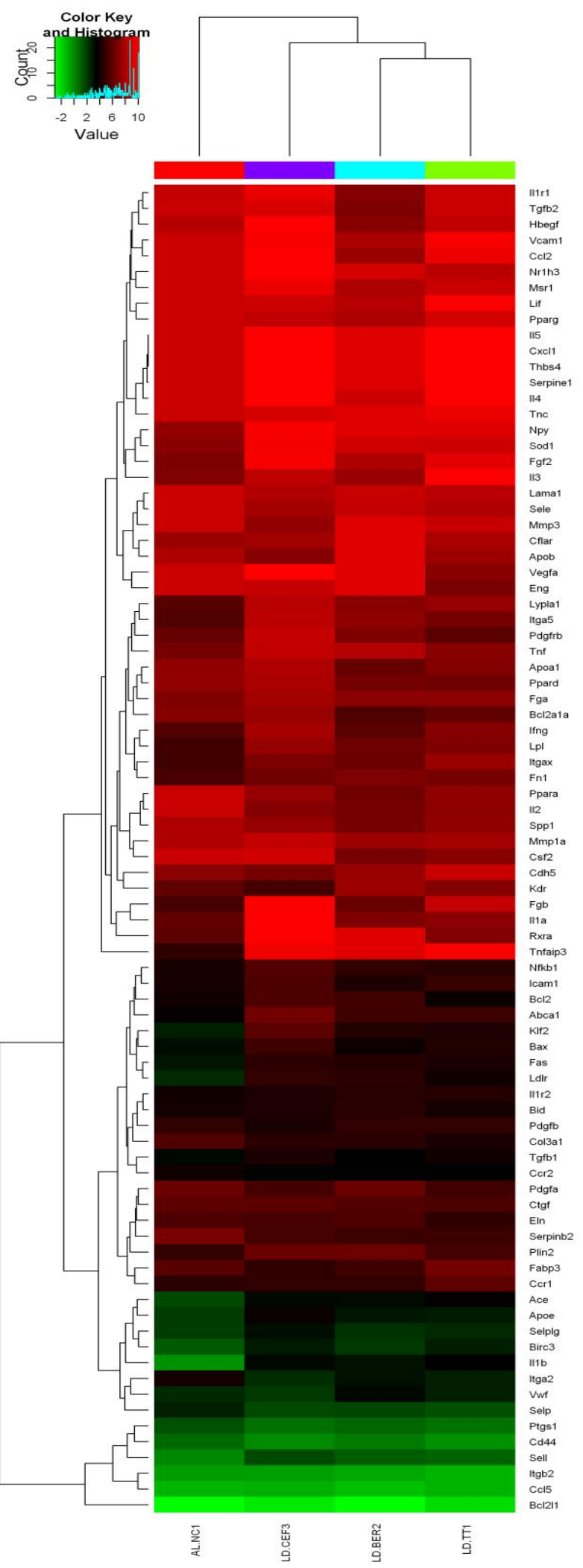

Figure 7. The inflammatory responses and atherosclerosis-related gene expression profiles among AL, CS, LD, BER, and CEF mice. (A) Microarray comparison of 84 atherosclerosis-related genes among the hepatic tissues of AL.NC1, CS.TT1, CS.BER2, and CS.CEF3. (B) Microarray comparison of 84 atherosclerosis-related genes among the hepatic tissues of AL.NC1, LD.TT1, LD.BER2, and LD.CEF3. 
CS Upregulates Atherosclerosis-Related Genes And BER Enhances CS-upregulated Atherogenesis-Related Gene Expression

To monitor the changes from atherogenesis to atherosclerosis upon CS/LD and antibiotic treatments, we quantified the expression levels of 84 atherosclerosis-related genes in $A L$, CS, LD, BER, and CEF mice (Additional file 2). As compared with AL.NC1 and considering two-fold changes, it was evident that eight genes are upregulated and 21 genes downregulated in CS.TT1 (Figure 7A), while 11 genes are upregulated and 29 genes downregulated in LD.TT1 (Figure 7B). It was noted that Eng, Itga2, Pdgfa and Serpinb2 are commonly upregulated, whereas Ace, Fgb, Fgf2, Ifng, II1a, II1b, Itgax, LdIr, Lpl, Npy, Sod1 and Tnfaip 3 are generally downregulated in CS.TT1 and LD.TT1. Interestingly, those downregulated genes encode pro-inflammatory cytokines, for example, Ifng, II1a, I/1b and Tnfaip3 encode IFN- - , IL-1 $\alpha$, IL-1 $\beta$ and TNF- $\alpha$.

In the CS group, treatment by BER leads to upregulation of 22 genes compared with AL.NC1 and upregulation of 29 genes compared with CS.TT1. In similar, treatment by CEF allows upregulation of 11 genes compared with AL.NC1 and upregulation of 16 genes compared with CS.TT1. In the LD group, 11 and eight genes are upregulated in LD.BER2 and LD.CEF3 compared with AL.NC1, while 19 and 23 genes are upregulated in LD.BER2 and LD.CEF3 compared with LD.TT1 (Table 3). Intriguingly, some pro-inflammatory cytokine, chemokine and corresponding receptor genes are upregulated after treatment by antibiotics. For example, Ccr1, Csf2, Ifng, II1a, II1r1 and I/2 are upregulated in CS.BER2, and Ccr1, Csf2, II1a, II1r1 and II2 are upregulated in CS.CEF3. In similar, Ccr1, Ifng, II1r1, II3, II4 and I/5 are upregulated in LD.BER2, and Ccr1, I/1b, I/1r2, I/2, I/3 and $/ 15$ are upregulated in LD.CEF3. These results implied antibiotic use enhances pro-inflammatory response, perhaps by inducing more severe gut dysbiosis.

Table 3. Comparison of atherosclerosis-related genes upregulated for two-fold and above in AL, CS, LD, BER, and CEF mice.

\begin{tabular}{|c|c|c|}
\hline Comparison & $\begin{array}{l}\text { Upregulated } \\
\text { Gene } \\
\text { number }\end{array}$ & Upregulated Gene Name \\
\hline CS.TT1 vs AL.NC1 & $\uparrow 8$ & Eng, II1r2, Itga2, Pdgfa, Ppard, Serpinb2, Spp1, Tgfb2 \\
\hline CS.BER2 vs AL.NC1 & $\uparrow 22$ & $\begin{array}{l}\text { Apoa1, Bcl2a1a, Ccr1, Ccr2, Cd44, Csf2, Eng, Il1r1, Il2, Itga2, } \\
\text { Kdr, Mmp3, Msr1, Pdgfa, Ppara, Ptgs1, Sele, Serpineb2, Spp1, } \\
\text { Tnc, Vcam1, Vwf }\end{array}$ \\
\hline CS.BER2 vs CS.TT1 & $\uparrow 29$ & $\begin{array}{l}\text { Ace, Apoa1, Bcl2a1a, Ccr2, Col3a1, Csf2, Ctgf, Eng, Fga, Fgb, } \\
\text { Hbegf, Ifng, II1a, II1r1, II2, Itga2, Itgax, Kdr, Ldlr, Lif, Lpl, Msr1, } \\
\text { Pdgfrb, Plin2, Ppara, Ptgs1, Sod1, Vcam1, Vwf }\end{array}$ \\
\hline CS.CEF3 vs AL.NC1 & $\uparrow 11$ & $\begin{array}{l}\text { Ccr1, Cd44, Col3a1, Csf2, Eng, II2, Itga2, Pdgfa, Ppara, Sele, } \\
\text { Spp1 }\end{array}$ \\
\hline CS.CEF3 vs CS.TT1 & $\uparrow 16$ & $\begin{array}{l}\text { Apoa1, Col3a1, Csf2, Cxcl1, Fgb, Hbegf, II1a, I/1r1, II2, Itgax, } \\
\text { Mmpa1,Pdgfrb, Plin2, Ppara, Sod1, Vcam1 }\end{array}$ \\
\hline LD.TT1 vs AL.NC1 & $\uparrow 11$ & $\begin{array}{l}\text { Cd44, Col3a1, Csf2, Eng, II2, Itga2, Pdgfa, Ppara, Selp, Serpinb2, } \\
\text { Vegfa }\end{array}$ \\
\hline
\end{tabular}


bioRxiv preprint doi: https://doi. org/10.1101/142588; this version posted May 27 2017. The copyright holder for this preprint (which was not certified by peer review) is the author/funder, who has granted bioRxiv a license to display the preprint in perpetuity. It is made available under aCC-BY-NC-ND 4.0 International license.

\begin{tabular}{|c|c|c|}
\hline LD.BER2 vs AL.NC1 & $\uparrow 11$ & $\begin{array}{l}\text { Apoa1, Bcl2a1a, Ccl2, Csf2, Hbegf, II1r1, II2, Ppara, Serpinb2, } \\
\text { Spp1, Tgfb2 }\end{array}$ \\
\hline LD.BER2 vs LD.TT1 & $\uparrow 19$ & $\begin{array}{l}\text { Bcl2/1, Ccl2, Ccr1,Cdh5, Cxcl1, Fabp3, Fgb, Fgf2, Hbegf, Ifng, } \\
\text { II1r1, II3, II4, II5, Itgax, Lif, Tgfb2, Vcam1, Serpine1 }\end{array}$ \\
\hline LD.CEF3 vs AL.NC1 & $\uparrow 8$ & Apob, Fabp3, II2, Itga2, Mmp3, Ppara, Selp, Serpineb2 \\
\hline LD.CEF3 vs LD.TT1 & $\uparrow 23$ & $\begin{array}{l}\text { Apob, Ace, Bcl2al1, Ccr1, Cdh5, Cflar, Fabp3, Kdr, Il1b, II1r2, I/2, } \\
\text { II3, II5, Itga2, Itgax, Lif, Mmp3, Pdgfb, Pparg, Sele, Tnc, Tnfaip3, } \\
\text { Vwf }\end{array}$ \\
\hline
\end{tabular}

Vcam1, upregulated by cytokines in CS.BER2, CS.CEF3 and LD.BER2, encodes a cell adhesion molecule and plays a role in the development of atherosclerosis and rheumatoid arthritis (Wu, 2007). Other frequently upregulated genes also include Apob, Apoa1, Ppara and Eng. A high level of ApoB was postulated to be the primary driver of plaques that cause vascular disease (atherosclerosis) (Tabas et al., 2007). ApoA1 is often used as a biomarker for prediction of cardiovascular diseases (McQueen et al., 2008). PPAR- $\alpha$ is a transcription factor and a major regulator of lipid metabolism in the liver (Staels et al., 2008). Endoglin is involved in the development of the cardiovascular system and in vascular remodeling (Li et al., 1999).

\section{CS Induces Steatogenesis And BER Enhances CS-Induced Steatohepatitis-Like Pathogenesis}

To reveal a putative association of animal-based diets with hepatic steatogenesis, we fed mice with $C S$ or LD and analyzed the characteristic fatty liver manifestation. It was noticed that the hepatic tissue of AL.NC1 (Figure 8A) exhibits less and small sized oil droplets than that of a CS.TT1 (Figure 8B). Intriguingly, it was also clarified that the more and larger sized hepatic oil droplets emerge in CS.BER2 (Figure 8C), whereas the less and smaller sized hepatic oil droplets occur in CS.CEF3 (Figure 8D). In the LD group, the numbers and volumes of oil droplets are only slightly increased in LD.TT1 (Figure 8E), but dramatically decreased in LD.BER2 (Figure 8F) or LD.CEF3 (Figure 8G). These results indicated BER use for one month can aggravate CS-induced hepatic steatogenesis, but CEF use for the same time only results in less advanced or even attenuated steatogenesis.

\section{A}

B

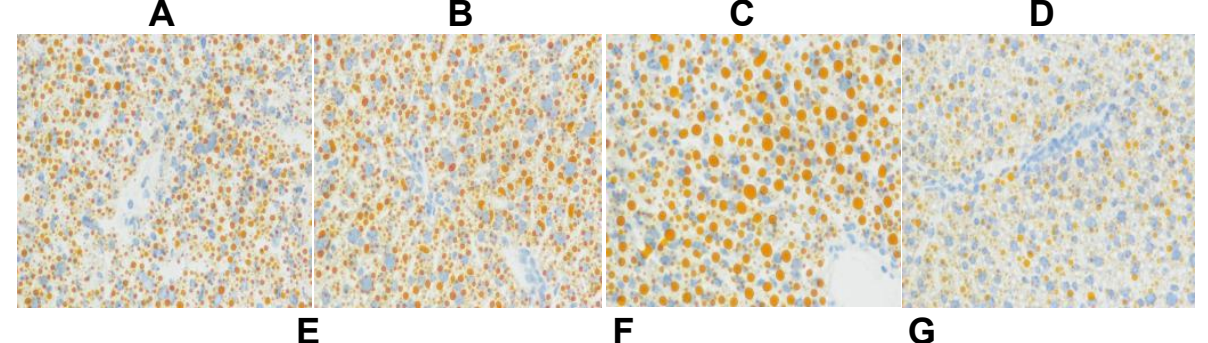

E

F

G

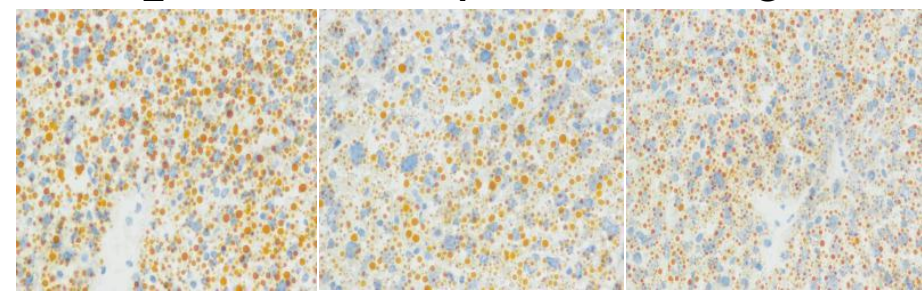


Figure 8. Hepatic steatogenesis and pro-/anti-inflammatory responses in the hepatic and adipose tissues of AL, CS, LD, BER, and CEF mice. (A)-(G) Oil red O staining in the hepatic tissue of AL.NC1 (A), CS.TT1 (B), CS.BER2 (C), CS.CEF3 (D), LD.TT1 (E), LD.BER2 (F), and LD.CEF3 (G), respectively $(\times$ 400).

Furthermore, we evaluated the potential risk of CS and CS+BER on NAFLD using 84 genes-comprising mouse fatty liver PCR microarray (Additional file 3). As compared with AL.NC1, while six NAFLD-related genes are upregulated in CS.TT1 with more than two-fold increases of mRNA levels, 12 NAFLD-related genes are upregulated in CS.BER2 with more than two-fold increases of mRNA levels (Table 4). For example, Serpine 1 mRNA involving in the adipokine signaling pathway is elevated for 15.06 folds in CS.TT1, and 14.83 folds in CS.BER2; Gsk mRNA involving in non-insulin dependent diabetes mellitus (NIDDM) is elevated for 3.16 folds in CS.TT1, and 15.67 folds in CS.BER2. Additionally, some upregulated genes in CS.BER2 are downregulated in CS.TT1. For example, Pdk4, Ldlr, and Ppard are downregulated in CS.TT1, but upregulated in CS.BER2. These results verified CS raises a risk of NAFLD, whereas BER further deteriorates such a severe condition.

Table 4 The hepatic expression levels of NAFLD-related genes with up/downregulation for more or less than two folds in AL.NC1, CS.TT1 and CS.BER2 mice

\begin{tabular}{|c|c|c|c|c|}
\hline \multirow[t]{3}{*}{ Pathway } & \multirow[t]{3}{*}{ Gene } & \multirow[t]{3}{*}{ Description } & \multicolumn{2}{|c|}{ Changed mRNA (Folds) } \\
\hline & & & CS.TT1 & CS.BER2 \\
\hline & & & IAL.NC1 & IAL.NC1 \\
\hline \multirow{3}{*}{$\begin{array}{l}\text { Inflammatory } \\
\text { Response }\end{array}$} & Cebpb & CCAAT/enhancer binding protein (C/EBP), beta & 1.64 & 2.95 \\
\hline & Foxa2 & Forkhead box A2 & 3.72 & 4.90 \\
\hline & Ifng & Interferon gamma & 1.46 & 3.32 \\
\hline \multirow{2}{*}{$\begin{array}{l}\text { Carbohydrate/ } \\
\text { Cholesterol }\end{array}$} & Pdk4 & Pyruvate dehydrogenase kinase, isoenzyme 4 & 0.77 & 2.72 \\
\hline & Apoe & Apolipoprotein E & 1.35 & 2.18 \\
\hline Metabolic & Ldlr & Low density lipoprotein receptor & 0.66 & 2.11 \\
\hline \multirow[t]{2}{*}{ Pathways } & Ppard & Peroxisome proliferator activator receptor delta & 0.71 & 2.01 \\
\hline & Srebf1 & Sterol regulatory element binding transcription factor 1 & 2.90 & 2.99 \\
\hline $\begin{array}{l}\text { Insulin } \\
\text { Signaling }\end{array}$ & Pik3r1 & $\begin{array}{l}\text { Phosphatidylinositol 3-kinase, regulatory subunit, } \\
\text { polypeptide } 1 \text { (p85 alpha) }\end{array}$ & 2.97 & 3.34 \\
\hline Pathway & Ptpn1 & Protein tyrosine phosphatase, non-receptor type 1 & 2.10 & 4.72 \\
\hline \multicolumn{5}{|l|}{ Adipokine } \\
\hline Signaling & Serpine1 & Serine (or cysteine) peptidase inhibitor, clade $\mathrm{E}$, member 1 & 15.06 & 14.83 \\
\hline \multicolumn{5}{|l|}{ Pathway } \\
\hline Genes & Gck & Glucokinase & 3.16 & 15.67 \\
\hline \multicolumn{5}{|l|}{ Involved in } \\
\hline NIDDM & & & & \\
\hline
\end{tabular}

\section{CS Elevates Dementia Biomarker Levels And BER boosts CS-Induced Dementia} Presentation

To evaluate the effects of CS/LD feeding and BER/CEF use on amyloidosis, we determined 
the level of amyloid $\beta$ peptide $(A \beta)$, a hallmark of Alzheimer's disease, in the cerebral tissues of all groups of mice. As results, the cerebral level of $A \beta$ is elevated in CS.TT1, and further elevated to a much higher level in CS.BER2 (Figure 9A). Accordingly, the cerebral level of tyrosine hydroxylase (Th), a rate-limiting enzyme for biosynthesis of the neurotransmitter dopamine, is declined in CS.TT1, and further declined to a much lower level in CS.BER2 (Figure 9B). These results indicated CS increases A $\beta$ and decreases TH, and BER confers a much higher $A \beta$ level and a much lower TH level, thereby implying BER use is a more risk factor than CS feeding in triggering dementia-like pathogenesis with amyloidosis and cognitive deficits.

It was observed that the A level in CS.CEF3 is lower than that in CS.BER2, whereas the Th level in CS.CEF3 is higher than that in CS.BER2, implying an augmented brain pathological alteration in CS.BER2 than in CS.CEF3 (Figure 9A and Figure 9B). On the other hand, elevation of the $A \beta$ level and decline of the Th level were not significantly noted in LD.TT1, LD.BER2 and LD-CEF3 (Figure 9A and Figure 9B), implying no remarkable alterations occurring in the brain structure and function after treatment by LD and/or BER/CEF. Furthermore, CS.BER2 show a low amount of the neurotransmitter acetylcholine (Ach), whereas CS.CEF3 show a high amount of Ach (Figure 9C). Interestingly, LD.BER2 and LD.CEF3 exhibit the higher amounts of Ach than LD.TT1. On the other hand, it was determined that activities of choline acetyltransferase (ChAT) responsible for Ach biosynthesis are almost unchanged among all tested mice (Figure 9D).

To explore the autophagic outcome upon treatments, we quantified microtubuleassociated proteins 1A/1B light chain 3B (LC3), the most widely used marker of autophygosomes, and compared the relative level of LC3-1, an unmodified form of LC3, and LC3-2, a lipid modified form of LC3 (Table 4). Consequently, CS.TT1 (lane 2 in Figure 9E), CS.CEF3 (lane 4 in Figure 9E), LD.BER2 (lane 6 in Figure 9E), and LD.CEF3 (lane 7 in Figure 9E) exhibit less LC3-1 and more LC3-2, whereas CS.BER2 (lane 3 in Figure 9E) and LD.TT1 (lane 5 in Figure 9E) show more LC3-1 and less LC3-2. These results suggested a higher autophagic activity (high-rate conversion from LC3-1 to LC3-2) in CS.TT1, CS.CEF3, LD.BER2 and LD.CEF, but a lower autophagic activity (low-rate conversion from LC3-1 to LC3-2) in CS.BER2 and LD.TT1, hence deciphering CS.BER2 possesses a high cerebral $A \beta$ level because it induces a low autophagic activity, and LD.TT1 possesses a low cerebral A $\beta$ level so it also induces a low autophagic activity.

A

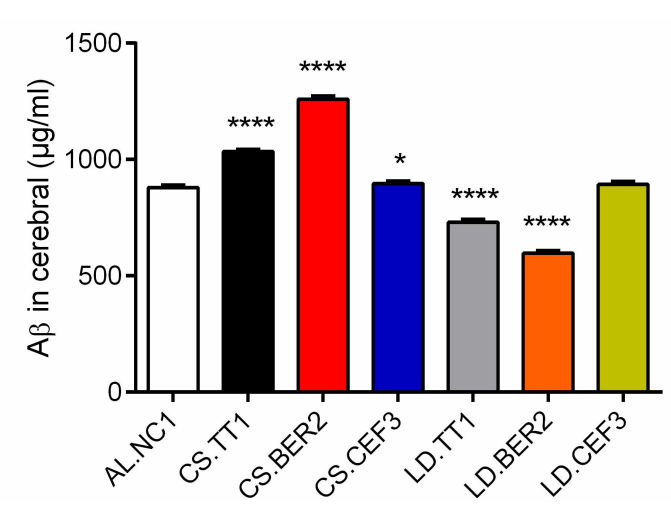

B

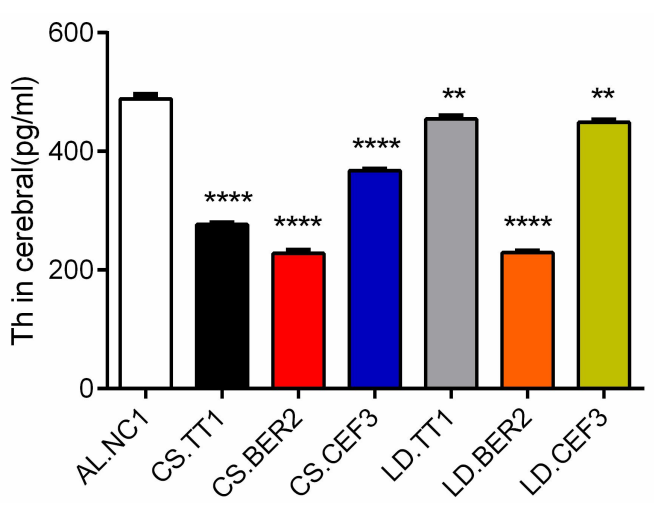


C

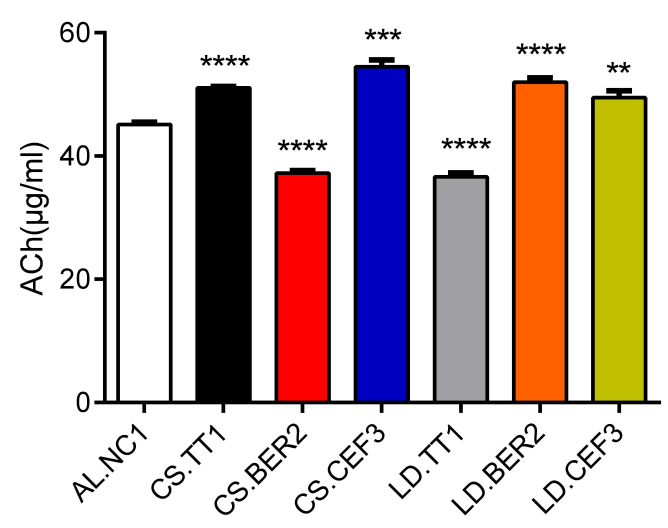

D

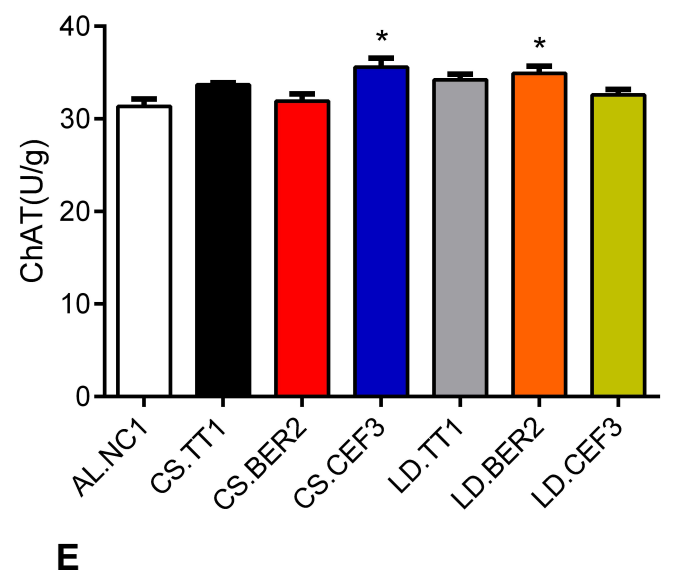

$\begin{array}{lllllll}1 & 2 & 3 & 4 & 5 & 6 & 7\end{array}$

LC3-2 LC3-1

Figure 9. The inflammatory responses and dementia-related gene expression profiles in $A L, C S$, LD, BER, and CEF mice. (A) A $\beta$ levels in the cerebral tissue. (B) Th levels in the cerebral tissue. (C) Ach levels in the cerebral tissue. (D) ChAT levels in the cerebral tissue. (E) LC3-2/-1 levels in the cerebral tissue, in which No.1-7 represent AL.NC1, CS.TT1, CS.BER2, CS.CEF3, LD.TT1, LD.BER2, and LD.CEF3, respectively.

Table 4 The relative gray scale values of LC3-1/2 in the hepatic or muscular tissues of AL, CS, LD, BER, and CEF mice.

\begin{tabular}{lrrrrrrr} 
& AL.NC1 & \multicolumn{1}{c}{ CS.TT1 } & CS.BER2 & CS.CEF3 & LD.TT1 & LD.BER2 & LD.CEF3 \\
\hline LC3-1/GAPDH (1) & 0.48 & 0.22 & 0.66 & 0.39 & 0.68 & 0.26 & 0.43 \\
LC3-2/GAPDH (2) & 0.42 & 0.35 & 0.50 & 0.47 & 0.34 & 0.64 & 0.49 \\
$1 / 2$ & 1.14 & 0.63 & 1.30 & 0.83 & 2.00 & 0.41 & 0.88 \\
\hline
\end{tabular}

Note: The ratios were calculated from the gray scale value of LC3-1/2 dividing that of GAPDH.

\section{DISCUSSION}

A putative association of gut opportunistic infection by commensal bacteria with neurodegenerative diseases was proposed (Li et al., 2016). The clinical manifestation of Parkinson's disease was shown to positively correlate with the abundance of Prevotellaceae, but negatively correlate with the abundance of Enterobacteriaceae (Scheperjans et al., 2015). It was demonstrated that oral administration of specific microbial metabolites to germ-free mice promotes neuroinflammation and motor neurodegenerative symptoms, whereas colonization of $\alpha$-synuclein-overexpressing mice with microbiota from Parkinson's disease-affected patients enhances physical impairments compared to microbiota transplants from healthy human donors (Sampson et al., 2016). It was revealed that LPS and K99 fimbriae protein (pilin) co-exist in the amyloid plaques of dementia brain tissues, suggesting a cerebral entry of bacterial components 
bioRxiv preprint doi: https://doi org/10.1101/142588; this version posted May 27, 2017. The copyright holder for this preprint (which was not certified by peer review) is the author/funder, who has granted bioRxiv a license to display the preprint in perpetuity. It is made available under aCC-BY-NC-ND 4.0 International license.

(Zhan, et al., 2016). Our study linked the dietary sulfate CS to the mucus-destroying bacteria SSB to the dementia hallmarks, including the high-level $A \beta$ and low-level Th and Ach in the brain. Additionally, we have previously found LPS-containing complete Freund's adjuvant can trigger brain pathological alterations by increasing $A \beta$ concentrations and decreasing Th activities (He et al., 2017).

A pathogenic mechanism underlying NAFLD or NASH (Brandl \& Schnable, 2017) remains unknown although the major risk factors are attributed to the obesity, diabetes and metabolic syndromes (Vuppalanchi et al., 2009). A potential tripartite relationship among the gut, diet, and liver diseases was recognized, a pivotal role of the individual gut microbiome on initiating NAFLD pathogenesis addressed, and animal- or plant-based diets accelerate NAFLD development by modulating the gut microbiome also highlighted (Mokhtari et al., 2017). More importantly, it was believed that sustained LPS leakage elicited by CS might not be completely neutralized by anti-LPS, thereby giving rise to a series of deleterious outcomes, such as induction of diabetes mellitus (Gomes et al., 2017). We found in this study that CS not only enhances adipose storage and impede mitochondrial functions in the liver, but also induces the expression of NAFLD/NIDDMrelated genes, implying an early hint of development toward NAFLD/NIDDM.

A correlation of high-fat diets with atherosclerosis was debating and controversial. For example, in a study involving rabbits fed the heated soybean oil, the gross initiation of atherosclerosis and marked induction of liver damage were histologically and clinically demonstrated (Greco \& Mingrone, 1990), but it was claimed that this outcome is not caused by dietary cholesterol rather than oxysterols or oxidized cholesterols (Kummerow, 2013). It was known that the dietary component that can be metabolized by the gut microbiota exerts a distinct effect on atherosclerosis, in which the dietary fibre and carnitine play a completely opposite role (Jonsson \& Backhed, 2017). Carnitine from red meat and phosphatidylcholine from egg yolk was verified to be converted to trimethylamine by the gut microbiota and subsequently oxidized to trimethylamine $\mathrm{N}$-oxide (TMAO) in the liver, eventually leading to atherosclerosis in the murine models (Wang et al., 2011, Koeth et al., 2013). Our study observed the upregulation of atherosclerosisrelated genes by CS feeding, accompanying with deficient and dysfunctional mitochondria.

It was worthy noting from this study that oral CS administration can dramatically change the multi-organ LPS and anti-LPS levels, in which an increase of the serum and muscular anti-LPS level and an accompanying decrease of the serum and muscular LPS level might urge some authors to draw a conclusion that CS exerts an anti-inflammatory effect and is apt to serve as a therapeutic agent for inflammatory diseases (Vergés et al., 2004; Clegg et al., 2006; Reginster et al., 2007). On the other hand, given CS really possesses a therapeutic value for amelioration of OA and IBD, it should only occur in the individuals without SSB, but not happen in the patients with SSB because CS enriches SSB, suggesting evaluation of CS in the inflammatory diseases should consider a potential effect of the gut microbiota.

Whether $A$. muciniphila is beneficial or harmful to human health remains another debating issue although our present work has concluded $A$. muciniphila with an abundance of $0.09 \%$ in CS.CEF3 or $0.3 \%$ in LD.CEF3 unlikely aggravates the 
steatohepatitis, atherosclerosis and dementia-like pathogenesis. In similar, other authors have shown antibiotics eliminating Akkermansia to reinforce the mucus barrier can block the heme-induced differential expression of oncogenes, tumor suppressors, and cell turnover genes, and prevent the heme-dependent cytotoxic micelles to reach the epithelium (Ijssennager et al., 2015). It was also evident that mucus-eroding microbiota including A. muciniphila and B. caccae can promote greater epithelial access and lethal colitis by the mucosal pathogen Citrobacter rodentium (Desai et al., 2016). However, A. muciniphila was proposed as a novel functional microbe with probiotic properties (Gomez-Gallego et al., 2016).

Indeed, mounting evidence is accumulated regarding the therapeutic values of A. muciniphila in metabolic diseases. For example, A. muciniphila is inversely correlated with obesity and type I diabetes in both mice and humans (Shin et al., 2014; Dao et al., 2016). The oral administration of $A$. muciniphila prevents a high-fat diet-induced obesity by altering adipose tissue metabolism and gut barrier function (Everard et al., 2013). The anti-obesity effect of a polyphenol-rich cranberry is associated with increased Akkermansia spp. population in the mouse gut (Anhê et al., 2015). Recently, a favorable effect of $A$. muciniphila on the improvement of glucose tolerance has been revealed upon maintaining a high IFNy level for controlling the multiple and primarily intracellular pathogen infections (Greer et al., 2016). Similarly, a positive correlation between A. muciniphila and gut health has been partially explained by its immune modulatory effects and immune tolerance to this commensal (Ottman et al., 2017).

As our opinion, there might be a threshold effect impacting the outcome of A. muciniphila, in which a minimal abundance of $A$. muciniphila might induce mucin production and promote mucus repair because it is anchored on the mucus and thus able to efficiently activate the mucus immunity to combating the gut opportunistic infection, but a maximal abundance of $A$. muciniphila should allow the excessive mucus opening and large-scale LPS leakage into the blood stream. In our present study, CEF was found to eradicate Desulfovibrio, but not to kill $A$. muciniphila, thereby exhibiting a less deleterious impact on the systemic pathogenesis under investigation. In contrast, we found BER use for one month can completely kill $A$. muciniphila, but cannot eradicate Desulfovibrio, showing an augmenting role on the systemic inflammatory pathogenesis. These results are well consistent with the popular concept of $A$. muciniphila as a probiotic beneficial to human health (Gomez-Gallego, et al., 2016).

BER as an antibacterial agent has been demonstrated to inhibits the growth of Staphylococcus aureus (Stermitz et al., 2000) and Microcystis aeruginosa (Zhang et al., 2010) when applied in vitro in combination with methoxyhydnocarpin, an inhibitor of multidrug resistance pumps. Some researchers have also explored the possible application of BER against the tractable infection by multi-resistant Staphylococcus aureus (MRSA) (Yu et al., 2005). Additionally, BER is under investigation to determine whether it may have applications as a drug in treating diabetes, hyperlipidemia, and cancer. Recently, BER has been proven to improve glucogenesis and lipid metabolism in NAFLD (Zhao et al., 2017), perhaps mainly acting on the visceral organs in addition to the gut microbiota per se. 
Overall, it is likely impossible to eradicate SSB/SRB from the gut by the monotherapy with a single antibiotic even a long-term use although CEF incidentally ameliorates CS/LD-driven systemic inflammatory disorders by nursing the beneficial probiotic bacterium. In such a context, a homeostatic gut symbions is a pivotal determinant for human gut health. Some studies have unraveled the diet, exercise and antiglycemic drugs may alter the development towards NFALD/NASH (Adams \& Angulo, 2006; Veena et al., 2014), but no pharmacological treatment regimens have been approved until 2015 (Ratziu et al., 2015). It is generally believed that a reasonable replacement of red meat by fruits and vegetables can impede the progression of atherosclerosis and decrease the risk of cardiovascular diseases (Wang et al., 2014). Our present study also addressed a fact that the meat diet rich in CS can evoke the systemic inflammatory pathogenesis by enriching mucus-destroying bacteria, strongly advising to restrict daily meat intake as early and frequently as possible.

\section{EXPERIMENTAL PROCEDURES}

\section{Animals, Diets And Antibiotic Uses}

The female Kunming mice (four-week old, 15-20 g) that belong to an out-bred population from SWISS mice were provided by The Experimental Animal Centre of Guangzhou University of Chinese Medicine in China (Certificate No. 44005800001448). All mice were housed on a 12-h light and 12-h dark cycle at $25^{\circ} \mathrm{C}$ with ad libitum chow and free water drinking. After one-week quarantine, mice were randomly divided into four groups: a control group of mice fed with ad libitum chow (AL.NC1), a model group of mice intragastrically fed with $250 \mu \mathrm{l} \mathrm{CS} \mathrm{(100} \mathrm{mg/ml,} \mathrm{FocusChem,} \mathrm{Shandong,} \mathrm{China)} \mathrm{daily} \mathrm{for} \mathrm{one} \mathrm{month}$ in addition to Ad libitum (CS.TT1), a model group of mice intragastrically fed with $200 \mu$ warm lard daily for one month in addition to Ad libitum (LD.TT1), a treatment group of CS mice administered BER via drinking water containing $0.1 \mathrm{~g} / \mathrm{ml}$ of berberine hydrochloride (CS.BER2) or containing $100 \mathrm{mg} / \mathrm{ml}$ of cefotaxime sodium (CS.CEF3) for one month, and a treated group of LD mice administered BER via drinking water containing $100 \mathrm{mg} / \mathrm{ml}$ of berberine hydrochloride (LD.BER2) or containing $100 \mathrm{mg} / \mathrm{ml}$ of cefotaxime sodium (LD.CEF3) for one month. Three to six mice were included within each group. All experiments were approved by The Animal Care Welfare Committee of Guangzhou University of Chinese Medicine (No. SPF-2015009). The experimental protocols complies with the requirements of animal ethics issued in the Guide for the Care and Use of Laboratory Animals of the NIH, USA.

\section{Gut Microbiota Metagenomic Analysis And Gene Expression Microarray}

The gut microbiota in a mouse fecal sample was identified by the high-throughput $16 \mathrm{~S} V \mathrm{X}$ sequencingbased classification procedure. The sequencing (sample preparation, DNA extraction and detection, amplicon purification, library construction and online sequencing) and data analysis (paired end-reads assembly and quality control, operational taxonomic units cluster and species annotation, alpha diversity and beta diversity) were conducted by Novogene (Beijing, China). The $\mathrm{RT}^{2}$ Profiler $^{\mathrm{TM}} \mathrm{PCR}$ Array Mouse Atherosclerosis ( PAMM-038Z ) and the RT ${ }^{2}$ Profiler ${ }^{\mathrm{TM}}$ PCR Array Mouse Fatty Liver ( PAMM-157Z ) were purchased from SABioscience Qiagen (Hilden, Germany). The experiment was performed by Kangchen Biotechnology (Shanghai, China).

\section{Western Blotting (WB) And Enzyme-Linked Immunosorbent Assay (ELISA)}


The reference protein GAPDH and all antigen proteins were immunoquantified by WB according to manufacture's manuals. The gray scale values were analyzed by the IMAGEJ software. Antibodies against JAK2, STAT3, AMPKa1, SIRT1, MFN2, UCP1 were purchased from Abcam (Cambridge, UK). ELISA kits for TNF- $\alpha$, LPS, anti-LPS, and TH were purchased from Chenglin Biotech (Beijing, China).

\section{Histochemical Analysis}

Cut fresh frozen tissue sections at 5-10 $\mu \mathrm{m}$ thick and mount on slides. Air dry slides for 30-60 min at room temperature and then fix in ice cold 10\% formalin for 5-10 min. Air dry again for another 30-60 min or rinse immediately in 3 changes of distilled water. Let slides air dry for a few min. Place in absolute propylene glycol for 2-5 min to avoid carrying water into oil red $\mathrm{O}$. Stain in pre-warmed $0.5 \%$ oil red O solution $\left(0.5 \mathrm{~g}\right.$ oil red $\mathrm{O}$ and $100 \mathrm{ml}$ propylene glycol) for $8-10 \mathrm{~min}$ in $60^{\circ} \mathrm{C}$ oven. Differentiate in $85 \%$ propylene glycol solution for 2-5 min. Rinse in 2 changes of distilled water. Stain in haematoxylin for 30 sec. Wash thoroughly in running tap water for $3 \mathrm{~min}$. Place slides in distilled water. Mount with glycerin jelly.

\section{Immunohistochemical Analysis}

The primary antibody against SLPI was purchased from Novus Biologicals (Littleton, USA), and the primary antibody against MUC1 was provided by CapitalBio (Beijing, China). Formalin-fixation, paraffin-embedment and deparaffinization were the same as HE staining procedure described above. Sections were incubated at room temperature with $3 \% \mathrm{H}_{2} \mathrm{O}_{2}$ to block endogenous peroxidase, and then repaired in boiling citric acid. After washing in phosphate-buffered solution, sections were blocked by $2 \%$ bovine serum albumin and incubated with $1: 100$ diluted primary antibodies at $37^{\circ} \mathrm{C}$ for $1 \mathrm{~h}$. After washing again, sections were incubated with biotinylated secondary antibodies at $37^{\circ} \mathrm{C}$ for $20 \mathrm{~min}$. After washing again, sections were incubated with diaminobenzidine for 1-5 min. After rinsing with tap water, sections were counter stained by haematoxylin. After completion of dehydration, clearance and mounting, photos were taken under the microscope (OLYMUPUS BX-51).

\section{Electronic Microscopy and Laser Con-focal Microscopy}

After treatment, cells were harvested and fixed in $2.5 \%$ glutaraldehyde in $0.1 \mathrm{M}$ phosphate buffer for $3 \mathrm{~h}$ at $4{ }^{\circ} \mathrm{C}$, followed by post-fixation in $1 \%$ osmium tetroxide for $1 \mathrm{~h}$. Samples were dehydrated in a graded series of ethanol baths, and infiltrated and embedded in Spurr's low-viscosity medium. Ultra-thin sections of $60 \mathrm{nM}$ were cut in a Leica microtome, double-stained with uranyl acetate and lead acetate, and examined in a Hitachi 7700 transmission electron microscope at an accelerating voltage of $60 \mathrm{kV}$.

The fresh samples of mouse skeletal muscles were fixed in paraformadehyde for $24 \mathrm{~h}$. After repeatedly rinsed, the fixed tissues were dehydrated by gradient ethanol. For embedding and sectioning, the tissue slices were pasted on the slides and coated at $50{ }^{\circ} \mathrm{C}$. After they were de-waxed by a transparent reagent and rinsed, the slides were incubated with antibodies and stained by 4',6-diamidino-2-phenylindole (DAPI). After drying, a fluorescence quencher was added and slides were sealed. The fluorescence-labeled second antibodies and the first antibody against MUC1 were purchased from Biosynthesis Biotechnology (Beijing, China). Immunoblotting was carried out based on the manufacturer's instructions.

\section{Spectrophotometry}

The reagent kits for measurement of ATP and LA were purchased from Jiancheng Biotech (Nanjing, China). All determination procedures were according to manufacturers' instructions. 


\section{Statistical Analysis}

The software SPSS 22.0 was employed to analyze data, and the software GraphPad Prism 5.0 was employed to plot graphs. The Independent Simple Test was used to compare all groups, but the Kruskal-Wallis Test followed by Nemenyi test was used when the data distribution is skewed. The significance level ( $p$ value) was set at $<0.05\left({ }^{*}\right),<0.01\left(^{* *}\right),<0.001\left(^{(* \star}\right)$ and $<0.0001{ }^{(* * *}$ ).

\section{SUPPLEMENTARY INFORMATION}

The datasets supporting the conclusions of this article are included in the supplementary information (Additional file 1 includes gut microbiota metagenomic data, Additional file 2 includes atherosclerosis-related gene microarray data, and Additional file $\mathbf{3}$ includes fatty liver-related gene microarray data).

\section{AUTHOR CONTRIBUTIONS}

QPZ, QW and QX designed the study. TL, YPC, and LLT carried out the experiments. XAH performed bioinformatics analysis. SQH, CQL, QW and QX participated in the interpretation of results. QPZ wrote the manuscript with input from other authors. All authors read and approved the final manuscript.

\section{ACKNOWLEDGMENTS}

This work was supported by the National Natural Science Foundation of China (No. 81273620 to Qing-Ping Zeng, No. 81673861 to Chang-Qing Li, and No. 81273817 and No. 81473740 to Qi Wang), and Guangdong Science and Technology Plan Project (No. 20150404042 to Qin Xu). We thank our colleagues in the Tropical Medicine Institute and Clinical Pharmacology Institute, Guangzhou University of Chinese Medicine, China. We also thank Kangchen Biotechnology Co, Shanghai, China for performance of RT-PCR microarray experiments, and Novogene, Beijing, China for conduction of gut microbiota metagenomic analysis.

\section{REFERENCES}

Aalvink, S., Skurnik, M., Boeren, S. Huuskonen, L., Aalvink, S., Skurnik, M., Boeren, S., et al. (2017). Pili-like proteins of Akkermansia muciniphila modulate host immune responses and gut barrier function. PLoS One. 12, e0173004.

Adams, L. A., and Angulo, P. (2006). Treatment of non-alcoholic fatty liver disease. Postgrad. Med. J. 82, 315-322.

Anhê, F.F., Roy, D., Pilon, G., Dudonné, S., Matamoros, S., Varin, T.V., Garofalo, C., Moine, Q., Desjardins, Y., Levy, E., et al. (2015). A polyphenol-rich cranberry extract protects from diet-induced obesity, insulin resistance and intestinal inflammation in association with increased Akkermansia spp. population in the gut microbiota of mice. Gut. $64,872-883$.

Attene-Ramos M S, Wagner E D, Gaskins H R, and Plewa, M.J. (2007) Hydrogen sulfide induces direct radical-associated DNA damage. Mol. Cancer Res. 5, 455-459. 
bioRxiv preprint doi: https://doi.org/10.1101/142588; this version posted May 27, 2017. The copyright holder for this preprint (which was not certified by peer review) is the author/funder, who has granted bioRxiv a license to display the preprint in perpetuity. It is made available under aCC-BY-NC-ND 4.0 International license.

Reichenbach, S., Sterchi, R., Scherer, M., Trelle, S., Bürgi, E., Bürgi, U., Dieppe, P.A., and Jüni, P. (2007). Meta-analysis: chondroitin for osteoarthritis of the knee or hip. Ann Intern Med. 146, 580-590.

Clauss, A., Lilja, H., Lundwall, Å. (2002). A locus on human chromosome 20 contains several genes expressing protease inhibitor domains with homology to whey acidic protein. Biochem. J. 368, $233-242$.

Clegg, D.O., Reda, D.J., Harris, C.L., Klein, M.A., O'Dell, J.R., Hooper, M.M., Bradley, J.D., Bingham, C.O. 3rd, Weisman, M.H., Jackson, C.G. et al. (2006). Glucosamine, chondroitin sulfate, and the two in combination for painful knee osteoarthritis. New Eng. J. Med. 354, 795-808.

Dao, M.C., Everard, A., Aron-Wisnewsky, J., Sokolovska, N., Prifti, E., Verger, E.O., Kayser, B.D., Levenez, F., Chilloux, J., Hoyles, L. et al. (2016). Akkermansia muciniphila and improved metabolic health during a dietary intervention in obesity: relationship with gut microbiome richness and ecology. Gut. $65,426-436$.

David, L.A., Maurice, C.F., Carmody, R.N., Gootenberg, D.B., Button, J.B., Wolfe, B.E., Ling, A.V., Devlin, A.S., Varma, Y., Fischbach, M.A., et al. (2014). Diet rapidly and reproducibly alters the human gut microbiome. Nature 505, 559-563.

Desai, M.S., Seekatz, A.M., Koropatkin, N.M., Kamada, N., Hickey, C.A., Wolter, M., Pudlo, N.A., Kitamoto, S., Terrapon, N., Muller, A., et al. (2016). A dietary fiber-deprived gut microbiota degrades the colonic mucus barrier and enhances pathogen susceptibility. Cell. 167, 1339-1353. e21.

Everard, A., Belzer, C., Geurts, L., Ouwerkerk, J. P., Druart, C., Bindels, L. B., Guiot, Y., Derrien, M., Muccioli, G.G. Delzenne, N.M., et al. (2013). Cross-talk between Akkermansia muciniphila and intestinal epithelium controls diet-induced obesity. Proc. Natl. Acad. Sci. U.S.A. 110, 9066-9071.

FDA. (2004). Letter Regarding the Relationship Between the Consumption of Glucosamine and/or Chondroitin Sulfate and a Reduced Risk of: Osteoarthritis; Osteoarthritis-related Joint Pain, Joint Tenderness, and Joint Swelling; Joint Degeneration; and Cartilage Deterioration (Docket No. 2004P-0059). Addressed to John W. Emford, Esq. William K. Hubbard. Oct. 7, 2004.

<http://www.fda.gov/food/ingredientspackaginglabeling/labelingnutrition/ucm073400.htm>

Garcia-Rios, A., Torres-Peña, J.D., Perez-Jimenez, F., and Perez-Martinez, P. (2017). Gut microbiota: a new marker of cardiovascular disease. Curr. Pharm. Des. Doi: 10.2174/1381612823666170317144853.

Gomes, J.M., Costa, J.A., and Alfenas, R.C. (2017). Metabolic endotoxemia and diabetes mellitus: a systematic review. Metabolism. 68, 133-144.

Greco, A.V., and Mingrone, G. (1990). Serum and biliary lipid pattern in rabbits feeding a diet enriched with unsaturated fatty acids. Exp. Pathol. 40, 19-33.

Greer, R.L., Dong, X., Moraes, A.C.F., Ryszard, A. Zielke, R.A., Fernandes, G.R., Peremyslova, E., Vasquez-Perez, S., Schoenborn, A.A., Gomes, E.P. et al. (2016). Akkermansia muciniphila mediates negative effects of IFNy on glucose metabolism. Nat. Commun. 7, 13329.

Guerrini, M., Beccati, D., Shriver, Z., Naggi, A., Viswanathan, K., Bisio, A., Capila, I., Lansing, C., Guglieri, S., Fraser, B. et al. (2008). Oversulfated chondroitin sulfate is a contaminant in heparin associated with adverse clinical events. Nat. Biotechnol. 26, 669-675. 
He, J., Liao, T., Zhong, G.X., Zhang, J.D., Chen, Y.P., Wang, Q., Zeng, Q.P. (2017). Alzheimer's disease-like early-phase brain pathogenesis: self-curing amelioration of neurodegeneration from pro-inflammatory 'wounding' to anti-inflammatory ‘healing'. Cur. Alzheimer Res. Doi: $10.2174 / 1567205014666170417111420$.

Holleran, G., Lopetuso, L.R., Ianiro, G., Pecere, S., Pizzoferrato, M., Petito, V., Graziani, C., Mc Namara, D., Gasbarrini, A., and Scaldaferri, F. (2017). Gut microbiota and inflammatory bowel disease: an update. Doi: 10.23736/S1121-421X.17.02386-8.

Ishioka, M., Miura, K., Minami, S., Shimura, Y., and Ohnishi, H. (2017). Altered gut microbiota composition and immune response in experimental steatohepatitis mouse models. Dig. Dis. Sci. 62, 396-406.

Jevseva, D.S., Brown, G.A., Jones, D.L., Matzkin, E.G., Manner, P.A., Mooar, P., Schousboe, J.T., Stovitz, S., Sanders, J.O., Bozic, K.J., et al. (2013). The American Academy of orthopaediac surgeons evidence-based guideline on: treatment of osteoarthritis of the knee, 2nd edition. J. Bone Joint Surg. Am. $95,1885-1886$.

Johansson, M.E.V., Sjövall, H., and Hansson, G.C. (2013). The gastrointestinal mucus system in health and disease. Gastroenterol. Hapatol. 10, 352-361.

Jonsson, A.L., and Bäckhed, F. (2017). Role of gut microbiota in atherosclerosis. Nat. Rev. Cardiol. 14, 79-87.

Kishimoto, K., Viswanathan, K., Ganguly, T., Elankumaran, S., Smith, S., Pelzer, K., Lansing, C., Sriranganathan, N., Zhao, G., Galcheva-Gargova, Z. et al. (2008). Contaminated heparin associated with adverse clinical events and activation of the contact system. New Engl. J. Med. 358, 2457-2467.

Koeth, R.A., Wang, Z., and Levison, B.S. (2013). Intestinal microbiota metabolism of L-carnitine, a nutrient in red meat, promotes atherosclerosis. Nat. Med. 19, 576-585.

Kovatcheva-Datchary, P., Nilsson, A., Akrami, R., Lee, Y.S., De Vadder, F., Arora, T., Hallen, A., Martens, E., Björck, I., and Bäckhed, F. (2015). Dietary fiber-induced improvement in glucose metabolism is associated with increased abundance of Prevotella. Cell Metab 22, 971-982.

Kummerow, F. (2013). Scientist, 98, challenges orthodoxy on causes of heart disease. <Medicalxpress. Com>.

Leschelle, X., Goubern, M., Andriamihaja, M., Blottière, H.M., Couplan, E., Gonzalez-Barroso, M.D., Petit, C., Pagniez, A., Chaumontet, C., Mignotte, B., et al. (2005). Adaptative metabolic response of human colonic epithelial cells to the adverse effects of the luminal compound sulfide. Biochim. Biophys. Acta (BBA)-General Subjects, 1725, 201-212.

Li, C.Q., Zheng, Q., Wang, Q., and Zeng, Q.P. (2016). Biotic/abiotic stress-driven Alzheimer's disease. Front. Cell. Neurosci. 10, 269.

Li, D.Y., Sorensen, L.K., Brooke, B.S., Urness, L.D., Davis, E.C., Taylor, D.G., Boak, B.B., and Wendel, D.P. (1999). Defective angiogenesis in mice lacking endoglin. Science. 284, 1534-1537. 
Li, F., Hao, X., Chen, Y., Bai, L., Gao, X., Lian, Z., Wei, H., Sun, R., and Tian, Z. (2017). The microbiota maintain homeostasis of liver-resident T-17 cells in a lipid antigen/CD1d-dependent manner. Nat. Commun. 7, 13839.

Li, X.Y., Zhao, Z.X., Huang, M., Feng, R., He, C.Y., Ma, C., Luo, S.H., Fu, J., Wen, B.Y., and Ren, L. (2015). Effect of berberine on promoting the excretion of cholesterol in high-fat diet-induced hyperlipidemic hamsters. J. Transl. Med. 13, 278.

Li Y., and Zuo, G.Y. (2010). Advances in studies on antimicrobial activities of alkaloids. Chin. Trad. Herb. Drugs. $41,1006-1014$.

Lippi, G., Danese, E., Mattiuzzi, C., and Favaloro, E.J. (2017). The intriguing link between the intestinal microbiota and cardiovascular disease. Semin. Thromb. Hemost. Doi: 10.1055/s-0036-1597903.

Liu, C.S., Zheng, Y.R., Zhang, Y.F., and Long, X.Y. (2016). Research progress on berberine with a special focus on its oral bioavailability. Fitoterapia. 109, 274-282.

McQueen, M.J., Hawken, S., Wang, X., Ounpuu, S., Sniderman, A., Probstfield, J., Steyn, K., Sanderson, J.E., Hasani, M., Volkova, E., et al. (2008). Lipids, lipoproteins, and apolipoproteins as risk markers of myocardial infarction in 52 countries (the INTERHEART study): a case-control study. Lancet. 372 ,

224-233.

Mokhtari, Z., Gibson, D.L., and Hekmatdoost, A. (2017). Nonalcoholic fatty liver disease, the gut microbiome, and diet. Adv. Nutr. 8, 240-252.

Ottman, N., Reunanen, J., Meijerink, M., Pietilä, T.E., Kainulainen, V., Klievink, J., Huuskonen, L., Aalvink, S., Skurnik, M., Boeren, S. et al. (2017). Pili-like proteins of Akkermansia muciniphila modulate host immune responses and gut barrier function. PLoS One. 12, e0173004.

Pirillo, A., and Catapano, A.L. (2015). Berberine, a plant alkaloid with lipid- and glucose-lowering properties: from in vitro evidence to clinical studies. Atherosclerosis. 243, 449-461.

Rajilić-Stojanović, M., Jonkers, D.M., Salonen, A., Hanevik, K., Raes, J., Jalanka, J., de Vos, W.M., Manichanh, C., Golic, N., Enck, P. et al. (2015). Intestinal microbiota and diet in IBS: causes, consequences, or epiphenomena?. Am. J. Gastroenterol. 110, 278-287.

Ratziu, V., Goodman, Z., and Sanyal, A. (2015). Current efforts and trends in the treatment of NASH. J. hepatol. 62, S65-75.

Reginster, J.Y., Heraud, F., Zegels, B., and Bruyere, O. (2007). Symptom and structure modifying properties of chondroitin sulfate in osteoarthritis. Mini Rev. Med. Chem. 7, 1051-1061.

Roman-Blas, J.A., Mediero, A., Tardio, L., Portal-Nunez, S., Gratal, P., Herrero-Beaumont, G., and Largo, R. (2017a). The combined therapy with chondroitin sulfate plus glucosamine sulfate or chondroitin sulfate plus glucosamine hydrochloride does not improve joint damage in an experimental model of knee osteoarthritis in rabbits. Eur. J. Pharmacol. 794, 8-14.

Roman-Blas, J.A., Castaneda, S., Sanchez-Pernaute, O., Largo, R., Herrero-Beaumont, G., and CS/GS Combined Therapy Study Group. (2017b). Combined treatment with chondroitin sulfate and glucosamine sulfate shows no superiority over placebo for reduction of joint pain and functional impairment in patients 
with knee osteoarthritis: a six-month multicenter, randomized, double-bland, placebo-controlled clinical trial. Arthritis Rheumatol. 69, 77-85.

Sampson, T., Debelius, J.W., Thron, T., Janssen, S., Shastri, G.G., Ilhan, Z.E., Challis, C., Schretter, C.E., Rocha, S., Gradinaru, V., et al. (2016). Gut microbiota regulates motor deficits and neuroinflammation in a morsel of Parkinson's disease. Cell. 167, 1469-1480.

Shang, Q., Yin, Y., Zhu, L., Li, G., Yu, G., and Wang, X. (2016). Degradation of chondroitin sulfate by the gut microbiota of Chinese individuals. Int J Biol Micromol. 86, 112-118.

Scheperjans, F., Aho, V., Pereira, P.A., Koskinen, K., Paulin, L., Pekkonen, E., Haapaniemi, E., Kaakkola, S., Eerola-Rautio, J., Pohja, M., et al. (2015). Gut microbiota are related to Parkinson's disease and clinical phenotype. Mov. Disord. 30, 350-358.

Segarra, S., Martinez-Subiela, S., Cerda-Cuellar, M., Martinez-Puig, D., Munoz-Prieto, A., Rodriguez-Franco, F., Rodriguez-Bertos, A., Allenspach, K., Velasco, A., and Ceron, J. (2016). Oral chondroitin sulfate and probiotics for the treatment of cannie inflammatory bowel disease: a randomized, controlled clinical trial. BMC Vet. Res.12, 49.

Shin, N.R., Lee, J.C., Lee, H.Y., Kim, M.S., Whon, T. W., Lee, M.S., and Bae, J.W. (2014). An increase in the Akkermansia spp. population induced by metformin treatment improves glucose homeostasis in diet-induced obese mice. Gut. 63, 727-735.

Singh, J.A., Noorbaloochi, S., MacDonald, R., and Maxwell, L.J. (2015). Chondroitin for osteoarthritis. Cochrane Data. Sys. Rev. 1, CD005614.

Spence, J.D. (2016). Intestinal microbiome and atherosclerosis. EBioMedicine.13, 17-18.

Staels, B., Maes, M., Zambon, A. (2008). Peroxisome fibrates and future PPARa agonists in the treatment of cardiovascular disease. Nat. Clin. Pract. Cardiovasc. Med. 5, 542-553.

Stermitz, F.R., Lorenz, P., Tawara, J.N., Zenewicz, L.A., and Lewis, K. (2000). Synergy in a medicinal plant: antimicrobial action of berberine potentiated by $5^{\prime}$-methoxyhydnocarpin, a multidrug pump inhibitor. Proc. Nat. Acad. Sci. USA. 97, 1433-1437.

Sun, R., Yang, N., Kong, B., Cao, B., Feng, D., Yu, X., Ge, C., Huang, J., Shen, J., Wang, P. et al. (2017). Orally administered berberine modulates hepatic lipid metabolism by altering microbial bile acid metabolism and the intestinal FXR signaling pathway. Mol. Pharmacol. 91, 110-122.

Tabas, I., Williams, K.J., Borén, J. (2007). Subendothelial lipoprotein retention as the initiating process in atherosclerosis: update and therapeutic implications. Circulation. 116, 1832-1844.

Veena, J., Muragundla, A., Sidgiddi, S., and Subramaniam, S. (2014). Non-alcoholic fatty liver disease: need for a balanced nutritional source. Br. J. Nutr. 112, 1858-1872.

Vergés, J., and Castañeda-Hernández, G. (2004). On the bioavailability of oral chondroitin sulfate formulations: proposed criteria for bioequivalence studies. Proc. West. Pharmacol. Soc. 47, 50-53.

Vuppalanchi, R., and Chalasani, N. (2009). Nonalcoholic fatty liver disease and nonalcoholic steatohepatitis: selected practical issues in their evaluation and management. Hepatology. 49, 306-317. 
Wang, X., Ouyang, Y., Liu, J., Zhu, M., Zhao, G., Bao, W., and Hu, F.B. (2014). Fruit and vegetable consumption and mortality from all causes, cardiovascular disease, and cancer: systematic review and dose-response meta-analysis of prospective cohort studies. BMJ (Clinical research ed.). 349, g4490.

Wang, Z., Klipfell, E., and Bennett, B.J. (2011). Gut flora metabolism of phosphatidylcholine promotes cardiovascular disease. Nature. 472, 57-63.

Wilson, A., McLean, C., and Kim, R.B. (2016). Trimethylamine-N-oxide: a link between the gut microbiome, bile acid metabolism, and atherosclerosis. Curr. Opin. Lipidol. 27, 148-154.

Wu, G.D., Chen, J., Hoffmann, C., Bittinger, K., Chen, Y.Y., Keilbaugh, S.A., Bewtra, M., Knights, D., Walters, W.A., Knight, R., et al. (2011). Linking long-term dietary patterns with gut microbial enterotypes. Science. 334, 105-108.

Wu, T.C. (2007). The role of vascular cell adhesion molecule-1 in tumor immune evasion. Cancer Res. 67, 6003-6006.

Yu, H.H., Kim, K.J., Cha, J.D., Kim, H.K., Lee, Y.E., Choi, N.Y., and You, Y.O. (2005). Antimicrobial activity of berberine alone and in combination with ampicillin or oxacillin against methicillin-resistant Staphylococcus aureus. J. Med. Food. 8, 454-461.

Zhan, X., Stamova, B., Jin, L.W., DeCarli, C., Phinney, B., and Sharp F.R. (2016). Gram-negative bacterial molecules associate with Alzheimer's disease pathology. Neurology. 87, 2324-32.

Zhang, S., Zhang, B., Xing, K., Zhang, X., Tian, X. and Dai, W. (2010). Inhibitory effects of golden thread (Coptis chinensis) and berberine on Microcystis aeruginosa. Wat. Sci. Technol. 61, 763-769.

Zhao, L., Cang, Z., Sun, H., Nie, X., Wang, N., and Lu, Y. (2017). Berberine improves glucogenesis and lipid metabolism in nonalcoholic fatty liver disease. BMC Endocr. Disord. 17, 13.

Zhou, D., Pan, Q., Xin, F.Z., Zhang, R.N, He, C.X., Chen, G.Y., Liu, C., Chen, Y.W., and Fan, J.G. (2017). Sodium butyrate attenuates high-fat diet-induced steatohepatitis in mice by improving gut microbiota and gastrointestinal barrier. World J. Gastroenterol. 23, 60-75. 